Supporting Information:

\title{
First Example of Organocatalytic Asymmetric Mannich Reaction between Aldimines of Glycinates and Sulphonyl Imines
}

Lei Wu, Guangxun Li, Migu He, Yingwei Wang, Gang Zhao* and Zhuo Tang*

\author{
Contents
}

1. Typical preparation of glycine imine schiff base .................................................... 3

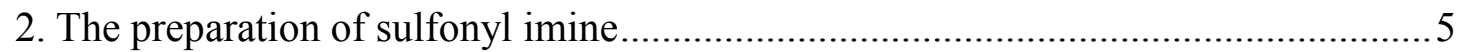

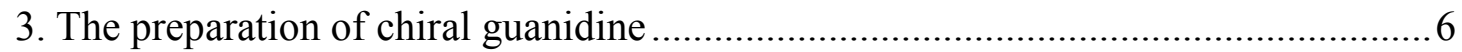

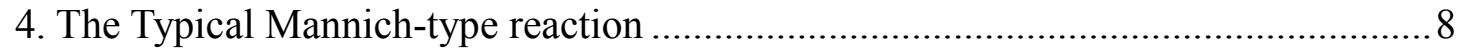

5. Generality explorations of the Mannich-type reaction …..................................... 12

6. Determination of absolute configuration for Mannich main adducts ...................... 16

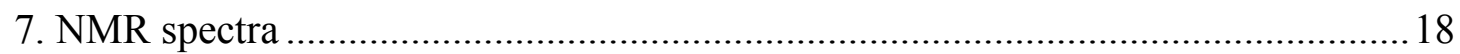




\section{General remarks}

All reactions were carried out with commercial available reagents. Analytical thin layer chromatography (TLC) was performed using Merck 60 F254 precoated silica gel plates with UV 254 indicator. Toluene, THF, EtOAc and $\mathrm{CH}_{2} \mathrm{Cl}_{2}$ was purchased as analytical pure. $\mathrm{CH}_{2} \mathrm{Cl}_{2}$ was dried with $\mathrm{NaH}$ under distilled condition. THF and Toluene was dried and distilled from sodium benzophenone. Melting points (m.p.) were measured on electrothermal digital melting point apparatus from Beijing Fukai Instruments Co.Ltd.

${ }^{1} \mathrm{H}$ NMR spectra were recorded on Bruker AMX 300 or $600 \mathrm{MHz}$ with $\mathrm{CDCl}_{3}$ or $\mathrm{CD}_{3} \mathrm{OD}$ as sovent. Chemical shifts were reported in ppm from $\mathrm{SiMe}_{4}(\delta 0.0)$ and the signal of $\mathrm{CDCl}_{3}(\delta 7.26$ singlet) and $\mathrm{CD}_{3} \mathrm{OD}\left(83.31\right.$ singlet). ${ }^{13} \mathrm{C}$ NMR spectra were recorded with $\mathrm{CDCl}_{3}$ and $\mathrm{CD}_{3} \mathrm{OD}$ as sovent. Chemical shifts were reported in ppm from $\mathrm{SiMe}_{4}(\delta 0.0)$ and the signal of $\mathrm{CDCl}_{3}(\delta 77.0$ triplet) and $\mathrm{CD}_{3} \mathrm{OD}(\delta 49.0$ singlet).

Enantiomeric excesses (ee) were determined by high performance liquid chromatogr aphy (HPLC) using corresponding commercial chiral column at $30{ }^{\circ} \mathrm{C}$ with Hatachidetector 254 or $210 \mathrm{~nm}$. Optical rotations were reported as follows: $[\alpha]_{\mathrm{D}}^{20}(\mathrm{c} \mathrm{g} / 100 \mathrm{~mL}$, in solvent).

${ }^{13} \mathrm{C}$ NMR spectra were collected on commercial instruments (100 or $150 \mathrm{MHz}$ ) with complete proton decoupling. Chemical shifts are reported in ppm from the tetramethylsilane with the solvent resonance as internal standard $\left(\mathrm{CDCl}_{3}, \delta=77.0, \mathrm{t}\right)$. High resolution mass spectrometry (HRMS) was measured on a commercial apparatus (ESI Source micrOTOF).

The racemic products used to determine the e.e. values were obtained by using 1,1,3,3-tetramethylguanidine (TMG) as catalyst. 
1. Typical preparation of glycine imine schiff base<smiles>[R]OC(=O)CN=Cc1cc([R])c([R])c([R])c1O</smiles>

Scheme S1. The synthesis of glycine imine schiff base

General Procedure for the Preparation of the glycine esters adlmines $\mathbf{4}$ according to previously report $^{[1]}$. To a solution of the corresponding amino acid ester chlorhydrate (30mmol, 1 equiv) in $\mathrm{CH}_{2} \mathrm{Cl}_{2}(45 \mathrm{~mL})$ were added $\mathrm{MgSO}_{4}$ and $\mathrm{NEt}_{3}$ ( $33 \mathrm{mmol}, 1.1$ equiv). The resulting mixture was stirred at the room temperature for $30 \mathrm{~min}$. Then the aldehyde ( $30 \mathrm{mmol}, 1$ equiv) was added, and the resulting mixture was stirred for $16 \mathrm{~h}$. Then the obtained sulution was filtered out and the organic layer washed with water, dried, and evaporated. The imines showed satisfactory NMR spectra. (Some of $\mathbf{4}$ were purified by base flash column chromatography).

\section{(E)-ethyl 2-((2-hydroxybenzylidene)amino)acetate(4a)}<smiles>CCOC(=O)C/N=C/c1ccccc1O</smiles>

$4 a$

According to the above procedure, afford 4a, light yellow oil; $90 \%$ yield;

${ }^{1} \mathrm{H}$ NMR $\left(600 \mathrm{MHz}, \mathrm{CDCl}_{3}\right) \delta 12.91(\mathrm{~s}, 1 \mathrm{H}), 8.37(\mathrm{~s}, 1 \mathrm{H})$, $7.38-7.30(\mathrm{~m}, 1 \mathrm{H}), 7.29-7.26(\mathrm{~m}, 1 \mathrm{H}), 6.98(\mathrm{~d}, J=8.3 \mathrm{~Hz}, 1 \mathrm{H})$, 6.89 (t, $J=7.4 \mathrm{~Hz}, 1 \mathrm{H}), 4.37$ (s, 2H), 4.25 (q, $J=7.1 \mathrm{~Hz}, 2 \mathrm{H})$,

$1.31(\mathrm{t}, J=7.1 \mathrm{~Hz}, 3 \mathrm{H})$.

${ }^{13} \mathrm{C}$ NMR $\left(151 \mathrm{MHz}, \mathrm{CDCl}_{3}\right) \delta 169.20,168.43,161.06,132.82,131.70,118.71,118.65$, $117.19,61.36,60.00,14.16$.

ESI-HRMS: calcd for $\mathrm{C}_{11} \mathrm{H}_{13} \mathrm{NO}_{3} \mathrm{H}^{+}\left(\left[\mathrm{M}+\mathrm{H}^{+}\right]\right)=208.0970$, Found 208.0969 .

\section{(E)-ethyl 2-((2-hydroxy-4-methoxybenzylidene)amino)acetate(4b)}<smiles>CCOC(=O)C/N=C/c1ccc(OC)cc1O</smiles>

light green solid, m.p. $75-75^{\circ} \mathrm{C}, 85 \%$ yield; ${ }^{1} \mathrm{H}$ NMR $\left(600 \mathrm{MHz}, \mathrm{CDCl}_{3}\right) \delta 13.37(\mathrm{~s}, 1 \mathrm{H}), 8.24(\mathrm{~s}, 1 \mathrm{H}), 7.14(\mathrm{~d}, J=$ $8.5 \mathrm{~Hz}, 1 \mathrm{H}), 6.65-6.19(\mathrm{~m}, 2 \mathrm{H}), 4.31(\mathrm{~s}, 2 \mathrm{H}), 4.23$ (q, $J=$ $7.2 \mathrm{~Hz}, 2 \mathrm{H}), 3.81(\mathrm{~s}, 3 \mathrm{H}), 1.30(\mathrm{t}, J=7.1 \mathrm{~Hz}, 3 \mathrm{H})$.

${ }^{13} \mathrm{C}$ NMR (151 MHz, DMSO-d6) $\delta$ 169.46, 167.39, 163.90, 163.67, 132.90, 112.50, 106.62, 101.12, 61.29, 59.42, 55.37, 14.16 .

ESI-HRMS: calcd for $\mathrm{C}_{12} \mathrm{H}_{15} \mathrm{NO}_{4} \mathrm{H}^{+}\left(\left[\mathrm{M}+\mathrm{H}^{+}\right]\right)=238.1074$, Found 238.1073 .

\section{(E)-tert-butyl 2-((3,5-dibromo-2-hydroxybenzylidene)amino)acetate(4c)}<smiles>CCOC(=O)C/N=C/c1cc(Br)cc(Br)c1O</smiles>

4c
Yellow oil, m.p. $50-53{ }^{\circ} \mathrm{C}$, yield $65 \%$.It was purified by flash chromatography on silica gel.

${ }^{1} \mathrm{H}$ NMR (600 MHz, $\left.\mathrm{CDCl}_{3}\right) \delta 13.95(\mathrm{~s}, 1 \mathrm{H}), 8.26(\mathrm{~s}$, 
1H), $7.70(\mathrm{t}, J=3.9 \mathrm{~Hz}, 1 \mathrm{H}), 7.34(\mathrm{~d}, J=2.2 \mathrm{~Hz}, 1 \mathrm{H}), 4.40(\mathrm{~s}, 2 \mathrm{H}), 4.30-4.20(\mathrm{~m}, 2 \mathrm{H}), 1.35-$ $1.27(\mathrm{~m}, 3 \mathrm{H})$.

${ }^{13} \mathrm{C}$ NMR $\left(151 \mathrm{MHz}, \mathrm{CDCl}_{3}\right) \delta 168.41,166.57,157.57,138.08,133.05,120.02,112.28$, 109.81, 61.68, 59.09, 14.16 .

ESI-HRMS: calcd for $\mathrm{C}_{12} \mathrm{H}_{15} \mathrm{NO}_{4} \mathrm{H}^{+}\left(\left[\mathrm{M}+\mathrm{H}^{+}\right]\right)=365.9169$, Found 365.9183

(E)-ethyl 2-((3,5-di-tert-butyl-2-hydroxybenzylidene)amino)acetate(4d)<smiles>CCOC(=O)C/N=C/c1cc(C(C)(C)C)cc(C(C)(C)C)c1O</smiles>

4d
Yellow solid; m.p. 39-43 ${ }^{\circ} \mathrm{C}$; yield $85 \%$;

${ }^{1} \mathrm{H}$ NMR (600 MHz, $\left.\mathrm{CDCl}_{3}\right) \delta 13.33(\mathrm{~s}, 1 \mathrm{H}), 8.39$ (s, $1 \mathrm{H}), 7.42(\mathrm{~d}, J=2.2 \mathrm{~Hz}, 1 \mathrm{H}), 7.11(\mathrm{~d}, J=2.3 \mathrm{~Hz}, 1 \mathrm{H}), 4.36$ (s, 2H), 4.25 (q, $J=7.1 \mathrm{~Hz}, 2 \mathrm{H}), 1.46$ (s, 9H), 1.32 (s, 9H).

${ }^{13} \mathrm{C}$ NMR $\left(151 \mathrm{MHz}, \mathrm{CDCl}_{3}\right) \delta 169.64,169.44,158.09$, 140.19, 136.88, 127.54, 126.29, 117.76, 61.27, 59.88, 35.07, 34.13, 31.49, 29.43, 14.18.

ESI-HRMS: calcd for $\mathrm{C}_{19} \mathrm{H}_{29} \mathrm{NO}_{3} \mathrm{H}^{+}\left(\left[\mathrm{M}+\mathrm{H}^{+}\right]\right)=320.2220$, Found 320.2220 .

\section{(E)-ethyl 2-((4-(diethylamino)-2-hydroxybenzylidene)amino)acetate(4e)}

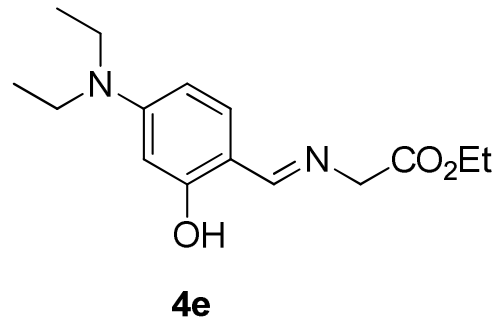

Light red solid; $90 \%$ yield; $53-55{ }^{\circ} \mathrm{C}$; Yield $=87 \%$;

${ }^{1} \mathrm{H}$ NMR $\left(600 \mathrm{MHz}, \mathrm{CDCl}_{3}\right) \delta 13.30(\mathrm{~s}, 1 \mathrm{H}), 8.11(\mathrm{~s}$, $1 \mathrm{H}), 7.03(\mathrm{~d}, J=8.7 \mathrm{~Hz}, 1 \mathrm{H}), 6.31-5.95(\mathrm{~m}, 2 \mathrm{H}), 4.47$ $-4.05(\mathrm{~m}, 4 \mathrm{H}), 3.36(\mathrm{q}, J=7.1 \mathrm{~Hz}, 4 \mathrm{H}), 1.23(\mathrm{dt}, J=$ 65.6, 7.1 Hz, 9H).

${ }^{13} \mathrm{C}$ NMR (151 MHz, DMSO-d6) $\delta$ 169.99, 166.69,

163.97, 151.51, 133.02, 108.42, 103.19, 97.99, 61.12, 59.36, 44.51, 14.18, 12.68.

ESI-HRMS: calcd for $\mathrm{C}_{15} \mathrm{H}_{22} \mathrm{~N}_{2} \mathrm{O}_{3} \mathrm{H}^{+}\left(\left[\mathrm{M}+\mathrm{H}^{+}\right]\right)=279.1703$, Found 279.1711 .

(E)-ethyl 2-((2,4-dihydroxybenzylidene)amino)acetate(4f)<smiles>CCOC(=O)C/N=C/c1ccc(O)cc1O</smiles>

Yellow solid; $128-131{ }^{\circ} \mathrm{C}$; $60 \%$ yield;

${ }^{1} \mathrm{HNMR}\left(600 \mathrm{MHz}, \mathrm{CDCl}_{3}\right) \delta 8.25(\mathrm{~s}, 1 \mathrm{H}), 7.13(\mathrm{~d}, J$ $=8.4 \mathrm{~Hz}, 1 \mathrm{H}), 6.40(\mathrm{~d}, J=2.2 \mathrm{~Hz}, 1 \mathrm{H}), 6.36(\mathrm{dd}, J=8.4$, $2.3 \mathrm{~Hz}, 1 \mathrm{H}), 4.32(\mathrm{~s}, 2 \mathrm{H}), 4.24$ (q, $J=7.1 \mathrm{~Hz}, 2 \mathrm{H}), 1.30$ (dd, $J=17.2,10.0 \mathrm{~Hz}, 3 \mathrm{H})$.

${ }^{13} \mathrm{C}$ NMR (151 MHz, DMSO-d6) $\delta$ 170.11, 168.30,

164.40, 162.50, 134.01, 111.77, 107.66, 103.01, 61.01, 58.23, 14.51.

ESI-HRMS: calcd for $\mathrm{C}_{15} \mathrm{H}_{22} \mathrm{~N}_{2} \mathrm{O}_{3} \mathrm{H}^{+}\left(\left[\mathrm{M}+\mathrm{H}^{+}\right]\right)=224.0917$, Found 224.0925 .

\section{(E)-methyl 2-((3,5-di-tert-butyl-2-hydroxybenzylidene)amino)acetate(4g)}<smiles>COC(=O)C/N=C/c1cc(C(C)(C)C)cc(C(C)(C)C)c1O</smiles>

$\mathbf{4 g}$ yellow oil; $35-38{ }^{\circ} \mathrm{C}$; yield $87 \%$;

${ }^{1} \mathrm{H}$ NMR $\left(600 \mathrm{MHz}, \mathrm{CDCl}_{3}\right) \delta 13.28(\mathrm{~s}, 1 \mathrm{H}), 8.39$ (s, $1 \mathrm{H}), 7.42(\mathrm{~d}, J=2.1 \mathrm{~Hz}, 1 \mathrm{H}), 7.11(\mathrm{~d}, J=2.2 \mathrm{~Hz}, 1 \mathrm{H})$, $4.38(\mathrm{~s}, 1 \mathrm{H}), 3.79(\mathrm{~s}, 1 \mathrm{H}), 1.46(\mathrm{~s}, 1 \mathrm{H}), 1.32(\mathrm{~s}, 1 \mathrm{H})$.

${ }^{13} \mathrm{C}$ NMR $\left(151 \mathrm{MHz}, \mathrm{CDCl}_{3}\right) \delta 169.87,169.77$,

$158.06,140.25,136.90,127.61,126.31,117.73,77.24,77.03,76.82,59.75,52.24,35.07,34.13$, 
31.48, 29.43 .

ESI-HRMS: calcd for $\mathrm{C}_{15} \mathrm{H}_{22} \mathrm{~N}_{2} \mathrm{O}_{3} \mathrm{H}^{+}\left(\left[\mathrm{M}+\mathrm{H}^{+}\right]\right)=306.2064$, Found 306.2064

(E)-tert-butyl 2-((3,5-di-tert-butyl-2-hydroxy benzy -lidene)amino)acetate(4h)<smiles>CC(C)(C)OC(=O)C/N=C/c1cc(C(C)(C)C)cc(C(C)(C)C)c1O</smiles>

$4 h$ yellow oil; $60-62{ }^{\circ} \mathrm{C}$; yield $79 \%$;

${ }^{1} \mathrm{H}$ NMR $\left(600 \mathrm{MHz}, \mathrm{CDCl}_{3}\right) \delta 13.45(\mathrm{~s}, 1 \mathrm{H}), 8.35$ (s, 1H), $7.40(\mathrm{~d}, J=2.1 \mathrm{~Hz}, 1 \mathrm{H}), 7.09(\mathrm{~d}, J=2.2 \mathrm{~Hz}$, $1 \mathrm{H}), 4.26(\mathrm{~s}, 2 \mathrm{H}), 1.50(\mathrm{~s}, 9 \mathrm{H}), 1.44(\mathrm{~s}, 9 \mathrm{H}), 1.30(\mathrm{~s}$, $9 \mathrm{H})$.

${ }^{13} \mathrm{C}$ NMR (151 MHz, MeOD) $\delta 169.21,168.63$, $158.15,140.05,136.84,127.37,126.19,117.79,81.81,60.52,35.05,34.11,31.48,29.41,28.08$. ESI-HRMS: calcd for $\mathrm{C}_{15} \mathrm{H}_{22} \mathrm{~N}_{2} \mathrm{O}_{3} \mathrm{H}^{+}\left(\left[\mathrm{M}+\mathrm{H}^{+}\right]\right)=348.2533$, Found 348.2534.

2. The preparation of sulfonyl imine ${ }^{[2]}$

$\mathrm{TiCl}_{4}(1.1 \mathrm{~mL}, 10 \mathrm{mmol}, 0.5$ quvic $)$ in dry $\mathrm{CH}_{2} \mathrm{Cl}_{2}(10 \mathrm{~mL})$ was added dropwise to a stirred, ice-cooled solution of benzaldehyde ( $20 \mathrm{mmol}, 1$ equiv), $p$-toluenesulfonamide ( $20 \mathrm{mmol}$ ), and $\mathrm{Et}_{3} \mathrm{~N}$ (7.1 g, $70 \mathrm{mmol}, 7$ equiv) in dry $\mathrm{CH}_{2} \mathrm{Cl}_{2}(40 \mathrm{~mL})$. The obtained mixture was stirred for 30 min. The titanium dioxide was removed by filtration through celite and washed with dichloromethane. Evaporation of the solvent under reduced pressure afforded a white solid which was broken up by refluxing in dry diethyl ether. The residual $\mathrm{Et}_{3} \mathrm{NHCl}$ was removed by filtration and the filtrate was concentrated to afford the crude imine. Purificaton by crystallization (hexane/dichloromethane) afforded 5a-5c.

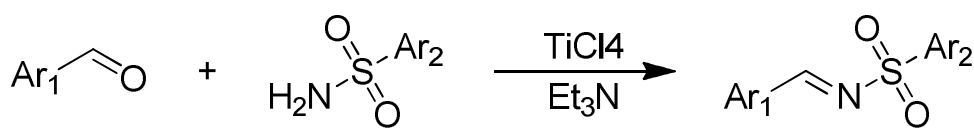

Scheme S2. The synthesis of sulfonyl imines 5a-5c.

The method for preparation of $\mathbf{5 d - 5 j}$ can be found in previously report ${ }^{[3]}$.<smiles>[R]C=NS(=O)(=O)c1cccc2cccnc12</smiles>

Scheme S3. The synthesis of sulfonyl imine $\mathbf{5 d - 5 i}$

(E)-N-benzylidene-2-methylbenzenesulfonamide (5b),<smiles>Cc1ccccc1S(=O)(=O)/N=C/c1ccccc1</smiles>

$5 b$

White solid; yield $72 \%$;

${ }^{1} \mathrm{H}$ NMR $\left(600 \mathrm{MHz}, \mathrm{CDCl}_{3}\right) \delta 9.10(\mathrm{~s}, 1 \mathrm{H}), 8.10(\mathrm{~d}, J=7.9$ $\mathrm{Hz}, 1 \mathrm{H}), 7.94(\mathrm{~d}, J=7.3 \mathrm{~Hz}, 2 \mathrm{H}), 7.62(\mathrm{t}, J=7.4 \mathrm{~Hz}, 1 \mathrm{H}), 7.50$ (t, $J=7.0 \mathrm{~Hz}, 3 \mathrm{H}), 7.35$ (dd, $J=16.4,7.8 \mathrm{~Hz}, 2 \mathrm{H}), 7.26(\mathrm{~s}, 1 \mathrm{H}), 2.75$ $(\mathrm{s}, 3 \mathrm{H})$. 
(E)-N-benzylidene-4-methoxybenzenesulfonamide (5c),<smiles>COc1ccc(S(=O)(=O)/N=C/c2ccccc2)cc1</smiles>

5c

White solid; yield $75 \%$;

${ }^{1} \mathrm{H}$ NMR $\left(600 \mathrm{MHz}, \mathrm{CDCl}_{3}\right) \delta 8.99(\mathrm{~s}, 1 \mathrm{H}), 7.91(\mathrm{dd}, J$ $=12.1,8.4 \mathrm{~Hz}, 4 \mathrm{H}), 7.59(\mathrm{t}, J=7.4 \mathrm{~Hz}, 1 \mathrm{H}), 7.47(\mathrm{t}, J=7.7$ $\mathrm{Hz}, 2 \mathrm{H}), 7.00$ (d, $J=8.9 \mathrm{~Hz}, 2 \mathrm{H}), 3.85$ (s, 3H).

${ }^{13} \mathrm{C}$ NMR (151 MHz, $\left.\mathrm{CDCl}_{3}\right) \delta 169.57,163.74,134.80$, $132.49,131.23,130.30,129.63,129.13,114.44,55.68$.

(E)-N-benzylidenequinoline-8-sulfonamide（5d）<smiles>O=S(=O)(/N=C/c1ccccc1)c1cccc2cccnc12</smiles>

$\mathbf{5 d}$

White solid; yield $47 \%$;

${ }^{1} \mathrm{H}$ NMR $\left(600 \mathrm{MHz}, \mathrm{CDCl}_{3}\right) \delta 9.58(\mathrm{~s}, 1 \mathrm{H}), 8.92(\mathrm{dd}, J=$ $4.2,1.6 \mathrm{~Hz}, 1 \mathrm{H}), 8.72(\mathrm{dd}, J=7.4,1.3 \mathrm{~Hz}, 1 \mathrm{H}), 8.20$ (dd, $J=$ $8.3,1.6 \mathrm{~Hz}, 1 \mathrm{H}), 8.10(\mathrm{dd}, J=8.2,1.1 \mathrm{~Hz}, 1 \mathrm{H}), 8.03-7.95$ (m, 2H), $7.71(\mathrm{t}, J=7.8 \mathrm{~Hz}, 1 \mathrm{H}), 7.62-7.57(\mathrm{~m}, 1 \mathrm{H}), 7.51-$ $7.38(\mathrm{~m}, 3 \mathrm{H})$.

${ }^{13} \mathrm{C}$ NMR $\left(151 \mathrm{MHz}, \mathrm{CDCl}_{3}\right) \delta 175.34,151.36,143.67,136.50,134.80,134.71,134.31$, $132.75,132.71,131.43,129.09,128.88,125.60,122.02,77.24,77.03,76.81$.

\section{The preparation of chiral guanidine}

General procedure: The method for preparation of $\mathbf{6 a - 6 h}$ following the above procedure can be found in previously report ${ }^{[4]}$.<smiles>[R]NC(=O)C1CCCN1</smiles>

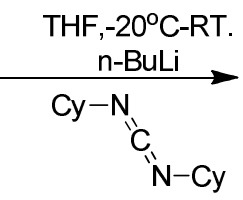<smiles>[R]N=C(O)C1CCCN1C(=O)NC1CCCCC1</smiles>

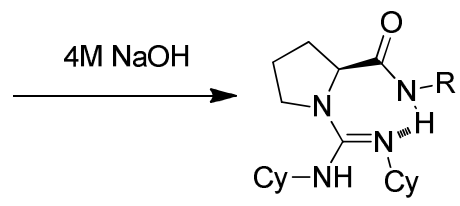

6a-6h

Scheme S4. The synthesis route of guanidine catalyst (6a-6h)

The preparation of (4R,5R)-4,5-diphenylimidazolidin-2-imine $6 \mathbf{i}$ according to previously literature ${ }^{[5]}$.<smiles>N=C1N[C@@H](c2ccccc2)[C@H](c2ccccc2)N1Cc1ccccc1</smiles>

$6 \mathbf{i}$

Scheme S5. The synthesis route of chiral guanidine $6 \mathbf{i}$

(1R,2R)-1,2-diphenylethane-1,2-diamine (1 g, $4.7 \mathrm{mmol})$ was dissolved in $\mathrm{MeCN}(5 \mathrm{~mL})$. The obtained solution was cooled to $0{ }^{\circ} \mathrm{C}$, and a $1 \mathrm{M}$ solution of cyanogen bromide in $\mathrm{MeCN}$ (4.7 $\mathrm{mL}, 4.7 \mathrm{mmol}$ ) was added dropwise via syringe. The mixture was equiped a reflux condenser and heated at reflux for $48 \mathrm{~h}$. The solution was concentrated and purified by column chromatography to afford white foam. The guanidinium salt was basified by dissolving in $\mathrm{CH} 2 \mathrm{Cl} 2$ 
and adding oil-free NaH. The suspension was shaken for thirty seconds, filtered through Celite, and concentrated to provide the guanidine $6 \mathrm{i}$ as a white powder in $51 \%$ yield.

${ }^{1} \mathrm{H} \mathrm{NMR}\left(600 \mathrm{MHz}, \mathrm{CDCl}_{3}\right) \delta 8.21(\mathrm{~s}, 1 \mathrm{H}), 8.12(\mathrm{~s}, 1 \mathrm{H}), 7.43-7.28(\mathrm{~m}, 3 \mathrm{H}), 7.19(\mathrm{~d}, J=7.3$ $\mathrm{Hz}, 2 \mathrm{H}), 4.73$ (s, 1H).

${ }^{13} \mathrm{C}$ NMR $\left(151 \mathrm{MHz}, \mathrm{CDCl}_{3}\right) \delta 160.38,140.57,128.87,128.23,126.43,70.31$.

ESI-HRMS: calcd for $\mathrm{C}_{15} \mathrm{H}_{15} \mathrm{~N}_{3}{ }^{+}\left[\mathrm{M}+\mathrm{H}^{+}\right]=238.1301$, found 238.1299 .

The preparation of 1,3-bis((S)-1-phenylethyl)imidazolidin-2-imine $\mathbf{6 j}$ an be found in previously report $^{[5]}$.

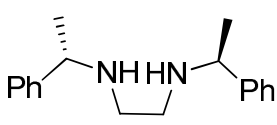

6j-a

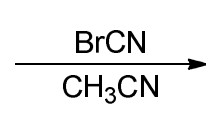<smiles>C[C@H](c1ccccc1)N1CCN([C@H](C)c2ccccc2)C1=N</smiles>

$6 \mathbf{j}$

Scheme S6. The synthesis route of chiral guanidine $6 \mathbf{j}^{[5]}$.

The method for preparation of $\mathbf{6 j} \mathbf{j}-\mathbf{a}$ can be found in previously literature ${ }^{[6]}$. $\mathbf{6 j}$ was Prepared as described for $\mathbf{6 i}$.

${ }^{1} \mathrm{H}$ NMR $\left(600 \mathrm{MHz}, \mathrm{CDCl}_{3}\right) \delta 7.45-7.30(\mathrm{~m}, 8 \mathrm{H}), 7.26(\mathrm{t}, J=6.9 \mathrm{~Hz}, 3 \mathrm{H}), 5.15(\mathrm{~s}, 2 \mathrm{H})$, $3.13(\mathrm{~d}, J=5.2 \mathrm{~Hz}, 2 \mathrm{H}), 2.92(\mathrm{~s}, 2 \mathrm{H}), 1.52(\mathrm{~d}, J=7.0 \mathrm{~Hz}, 6 \mathrm{H})$.

The preparation of $\mathrm{N}-((4 \mathrm{R}, 5 \mathrm{R})-1,3$-dimethyl-4,5-diphenylimidazolidin-2-ylidene)-1-pheny lmethanamine $6 \mathbf{k}^{[1]}$.

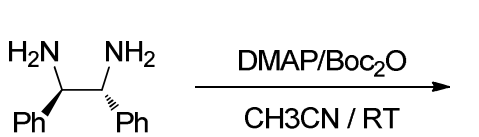

$6 k-a$<smiles>O=C1NC(c2ccccc2)C(c2ccccc2)N1</smiles>

6k-b<smiles>CN1C(=O)N(C)C(c2ccccc2)C1c1ccccc1</smiles>

$6 k-c$<smiles></smiles>

6k-d

6k

Scheme S7. The synthesis route of chiral guanidine $6 \mathbf{k}$.

The method for preparation of $\mathbf{6 k - c}$ can be found in previously report ${ }^{[7]}$.

A solution of 6k-c (3.0 g, $11.3 \mathrm{mmol})$ and oxalyl chloride $(1.6 \mathrm{~g}, 12.4 \mathrm{mmol})$ in anhydrous toluene $(100 \mathrm{ml})$ was refluxed for $12.5 \mathrm{~h}$. After cooling, the precipitate was filtered, repeatedly washed with anhydrous toluene, and dried under reduced pressure to give $\mathbf{6 k - d}$ as colorless solid. The crude solid was used for next step without further purification.

To a solution of a benzylamine $(0.33 \mathrm{~g}, 3.1 \mathrm{mmol})$ and $\mathrm{Et}_{3} \mathrm{~N}(0.66 \mathrm{mg}, 6.5 \mathrm{mmol})$ in $\mathrm{CH}_{2} \mathrm{Cl}_{2}$ (30 ml) was added dropwise a solution of $\mathbf{6 k - d}(1 \mathrm{~g})$ in $\mathrm{CH}_{2} \mathrm{Cl}_{2}(15 \mathrm{ml})$ at $\mathrm{rt}$. The mixture was 
stirred at RT for 1 hour and poured into dil. $\mathrm{HCl}$, and extracted with dichloromethane. The organic solution was evaporated to dryness. The residue was dissolved in water and washed with toluene. The aqueous solution was made alkaline with dil. aq. $\mathrm{NaOH}$ and extracted with $\mathrm{CH}_{2} \mathrm{Cl}_{2}$. The obtained organic solution was dried and evaporated to yield pure guanidines $6 \mathbf{k}$ as white solid.

${ }^{1} \mathrm{H}$ NMR $\left(600 \mathrm{MHz}, \mathrm{CDCl}_{3}\right) \delta 10.73(\mathrm{~s}, 1 \mathrm{H}), 7.48(\mathrm{~d}, J=7.3 \mathrm{~Hz}, 2 \mathrm{H}), 7.41(\mathrm{~d}, J=7.3 \mathrm{~Hz}$, 2H), $7.36(\mathrm{dd}, J=15.0,7.3 \mathrm{~Hz}, 7 \mathrm{H}), 6.96(\mathrm{~d}, J=6.6 \mathrm{~Hz}, 4 \mathrm{H}), 5.03(\mathrm{~s}, 1 \mathrm{H}), 4.81$ (d, $J=13.8 \mathrm{~Hz}$, $1 \mathrm{H}), 4.29(\mathrm{~d}, J=52.2 \mathrm{~Hz}, 2 \mathrm{H}), 3.11(\mathrm{~s}, 6 \mathrm{H})$.

The preparation of chiral di-guanidine catalyst $\mathbf{6} \mathbf{1}$ was found in previously literature ${ }^{[8]}$.
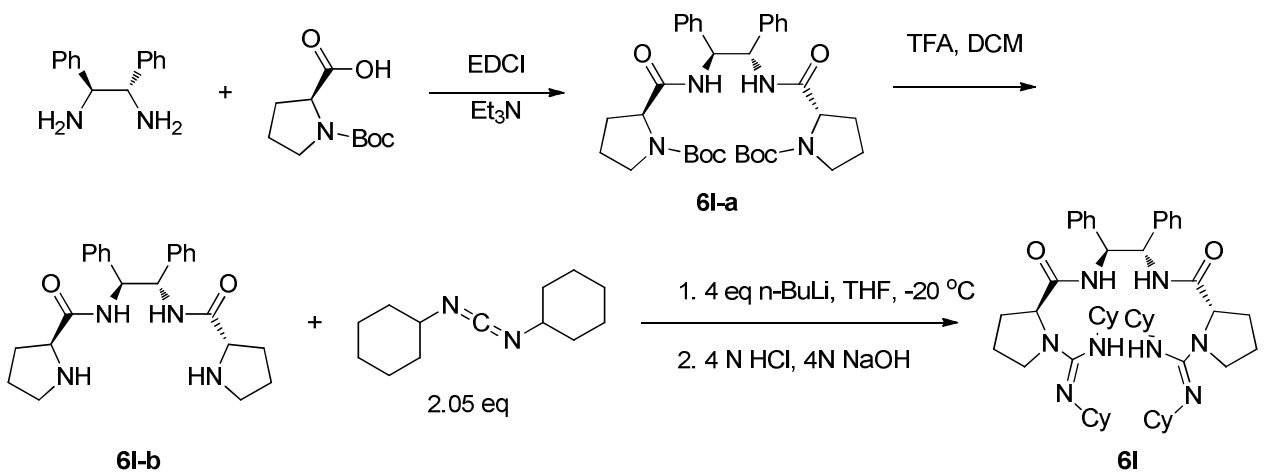

Scheme S8. The synthesis route of chiral guanidine $6 \mathbf{l}$

Product 61 was obtained following the literature procedure, matching the previously published characterization data.

${ }^{1} \mathrm{H}$ NMR $(600 \mathrm{MHz}, \mathrm{CDCl} 3) \delta 7.08(\mathrm{t}, \mathrm{J}=33.6 \mathrm{~Hz}, 10 \mathrm{H}), 5.16(\mathrm{~s}, 2 \mathrm{H}), 4.67(\mathrm{~s}, 2 \mathrm{H}), 3.25(\mathrm{~d}, \mathrm{~J}=$ $55.0 \mathrm{~Hz}, 2 \mathrm{H}), 2.92(\mathrm{~d}, \mathrm{~J}=32.0 \mathrm{~Hz}, 2 \mathrm{H}), 2.25(\mathrm{~d}, \mathrm{~J}=45.7 \mathrm{~Hz}, 1 \mathrm{H}), 1.95-1.46(\mathrm{~m}, 14 \mathrm{H}), 1.44-$ $0.81(\mathrm{~m}, 12 \mathrm{H})$.

\section{The Typical Mannich-type reaction}

Table S1. Examination of condition effect for Mannich-type reaction

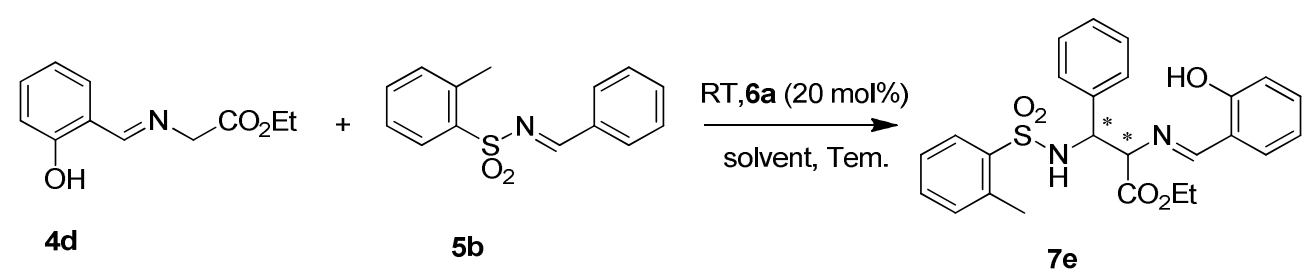

\begin{tabular}{lllllll}
\hline Entry $^{\mathrm{a}}$ & Tem. $\left[{ }^{\circ} \mathrm{C}\right]$ & Solvent & $\mathrm{X}[\mathrm{mol} \%]$ & ${\text { Yield}[\%]^{\mathrm{b}}}$ & $\mathrm{dr}^{\mathrm{c}}$ & $\mathrm{ee}[\%]^{\mathrm{d}}$ \\
\hline 1 & 10 & Toluene & 20 & 86 & $1.9: 1$ & $5 / 3$ \\
2 & 0 & Toluene & 20 & 85 & $2: 1$ & $7 / 2$ \\
3 & -10 & Toluene & 20 & 56 & $2: 1$ & $6 / 5$ \\
\hline
\end{tabular}




\begin{tabular}{lllllll}
\hline 4 & 0 & Ethyl ether & 20 & 90 & $1.2: 1$ & $0 / 4$ \\
5 & 0 & $\mathrm{THF}$ & 20 & 58 & $3: 1$ & $3 / 0$ \\
6 & 0 & $\mathrm{CH}_{2} \mathrm{Cl}_{2}$ & 20 & 56 & $13: 1$ & $4 / 5$ \\
7 & 0 & $\mathrm{CHCl}_{3}$ & 20 & 50 & $2.5: 1$ & $3 / 0$ \\
8 & 0 & $\mathrm{EtOH}$ & 20 & 92 & $1: 1$ & $0 / 0$ \\
9 & 0 & $\mathrm{CCl}_{4}$ & 20 & 86 & $17: 1$ & $0 / 4$ \\
10 & 0 & $\mathrm{CH}_{3} \mathrm{CN}$ & 20 & 75 & $16: 1$ & $0 / 0$ \\
$\mathbf{1 1}$ & $\mathbf{0}$ & $\mathbf{E A}$ & $\mathbf{2 0}$ & $\mathbf{9 0}$ & $\mathbf{1 . 7 : 1}$ & $\mathbf{1 1 / 2}$ \\
$\mathbf{1 2}$ & $\mathbf{0}$ & $\mathbf{E A}$ & $\mathbf{1 5}$ & $\mathbf{8 7}$ & $\mathbf{1 . 7 : 1}$ & $\mathbf{1 1 / 5}$ \\
13 & 0 & $\mathrm{EA}$ & 10 & 68 & $1.6: 1$ & $11 / 6$ \\
\hline
\end{tabular}

[a] Unless otherwise noted, the reaction was carried out with $0.06 \mathrm{mmol} \quad \mathbf{4 d}, 0.066 \mathrm{mmol} \mathbf{5 b}, 40$ mg $4 \AA$ and catalyst in solvent $(1.0 \mathrm{~mL})$ for $20 \mathrm{~h}$. [b] Yield of isolated product. [c] Determined by chiral HPLC. [d] Corresponding ee of anti/syn.

The Typical Mannich-type reaction.<smiles>CCOC(=O)CN=Cc1cc(C(C)(C)C)cc(Br)c1O</smiles>

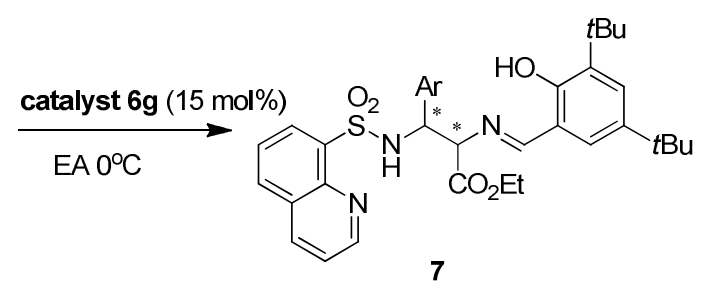

Scheme S9. The Typical procedure of Mannich-type reaction

To a $10 \mathrm{~mL}$ tube with a magnetic stirring bar were sequentially added the aldimines of glycine imine schiff base $\mathbf{4 d}$ ( $0.06 \mathrm{mmol}, 1.0$ equiv), sulfonyl imine $\mathbf{5}$ ( $0.066 \mathrm{mmol}, 1.1$ equiv), $40 \mathrm{mg} 4 \mathrm{~A}$ molecular sieve and $15 \mathrm{~mol} \%$ of catalyst $6 \mathrm{~g}$ in $1.5 \mathrm{~mL}$ ice EA under dry nitrogen at $0^{\circ} \mathrm{C}$ for $12 \mathrm{~h}$.. When the reaction completed (monitored by TLC), the obtained mixture was purified directly by flash chromatography on silica gel to afford 7 for H-NMR (confirmed the dr). The ee of the major isomer was determined by chiral HPLC analysis. (Note: The dr of some 7 was determined by HPLC analysis.)

(E)-ethyl 2-((3,5-dibromo-2-hydroxybenzylidene)amino)-3-(2-methylphenylsulfonamido)-3-phenylpropanoate (7b)<smiles>CCOC(=O)/C(=N/c1ccccc1O)C(N[S+](=O)c1ccccc1C)c1ccccc1</smiles>

7b
Yellow oil; $84 \%$ yield;

${ }^{1} \mathrm{H}$ NMR $\left(600 \mathrm{MHz}, \mathrm{CDCl}_{3}\right) \delta 12.46(\mathrm{~s}, 1 \mathrm{H}), 12.36$ $-12.25(\mathrm{~m}, 0.3 \mathrm{H}), 8.14(\mathrm{~s}, 1 \mathrm{H}), 7.99(\mathrm{~s}, 0.3 \mathrm{H}), 7.89(\mathrm{~d}, J$ $=8.0 \mathrm{~Hz}, 1 \mathrm{H}), 7.79(\mathrm{~d}, J=7.1 \mathrm{~Hz}, 0.3 \mathrm{H}), 7.32(\mathrm{dt}, J=$ $26.1,8.5 \mathrm{~Hz}, 2.8 \mathrm{H}), 7.23-7.04(\mathrm{~m}, 10 \mathrm{H}), 6.96(\mathrm{t}, J=$ $7.9 \mathrm{~Hz}, 1.4 \mathrm{H}), 6.91-6.84(\mathrm{~m}, 1.3 \mathrm{H}), 5.65(\mathrm{~d}, J=8.5 \mathrm{~Hz}$,

$0.3 \mathrm{H}), 5.38(\mathrm{~d}, J=8.2 \mathrm{~Hz}, 1 \mathrm{H}), 4.94(\mathrm{dd}, J=19.4,13.8 \mathrm{~Hz}, 1.4 \mathrm{H}), 4.39(\mathrm{~d}, J=5.5 \mathrm{~Hz}, 1 \mathrm{H}), 4.19$ (d, $J=4.7 \mathrm{~Hz}, 0.4 \mathrm{H}), 4.10-3.99(\mathrm{~m}, 2 \mathrm{H}), 3.95$ (d, $J=7.2 \mathrm{~Hz}, 0.4 \mathrm{H}), 2.54(\mathrm{~s}, 1 \mathrm{H}), 2.42(\mathrm{~s}, 2.8 \mathrm{H})$, 
$1.11(\mathrm{dt}, \mathrm{J}=14.2,7.1 \mathrm{~Hz}, 4 \mathrm{H})$.

${ }^{13} \mathrm{C}$ NMR $(151 \mathrm{MHz}, \mathrm{CDCl} 3) \delta 169.12,168.85,160.92,137.97,136.89,136.21,133.33$, 132.76, 132.44, 132.26, 129.38, 128.56, 128.42, 127.31, 127.08, 126.06, 118.91, 118.39, 117.19, 75.20, 61.77, 59.66, 20.17, 13.88 .

ESI-HRMS: calcd for $\mathrm{C}_{25} \mathrm{H}_{26} \mathrm{~N}_{2} \mathrm{O}_{5} \mathrm{SH}^{+}\left(\left[\mathrm{M}+\mathrm{H}^{+}\right]\right)=465.1490$, Found 465.1489

(E)-ethyl 2-((2-hydroxy-4-methoxybenzylidene)amino)-3-(2-methylphenylsulfona -mido) -3-phenylpropanoate (7c)

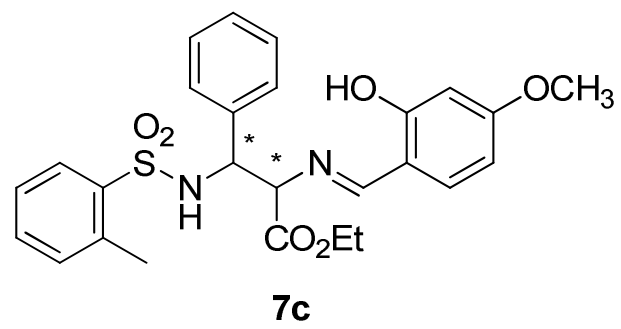

Yellow oil; 90\% yield;

${ }^{1} \mathrm{H}$ NMR $\left(600 \mathrm{MHz}, \mathrm{CDCl}_{3}\right) \delta 12.90(\mathrm{~s}$, $1 \mathrm{H}), 8.01(\mathrm{~d}, J=7.8 \mathrm{~Hz}, 2 \mathrm{H}), 7.88(\mathrm{~d}, J=7.8 \mathrm{~Hz}$, 1H), $7.46(\mathrm{t}, J=7.5 \mathrm{~Hz}, 0.8 \mathrm{H}), 7.32(\mathrm{t}, J=7.6$ $\mathrm{Hz}, 2.6 \mathrm{H}), 7.24-7.15$ (m, 4H), $7.12-7.04$ (m, $4 \mathrm{H}), 6.43(\mathrm{~d}, J=8.9 \mathrm{~Hz}, 2 \mathrm{H}), 5.47(\mathrm{~d}, J=8.4 \mathrm{~Hz}$, 1H), 4.92 (dd, $J=8.2,5.8 \mathrm{~Hz}, 1 \mathrm{H}), 4.87$ (d, $J=36.3 \mathrm{~Hz}, 1.6 \mathrm{H}), 4.33$ (d, $J=5.6 \mathrm{~Hz}, 1 \mathrm{H}), 4.05$ (ddd, $J=15.4,12.1,10.9 \mathrm{~Hz}, 3 \mathrm{H}), 3.82(\mathrm{~s}, 3 \mathrm{H}), 2.69$ (s, 2.4H), $2.43(\mathrm{~s}, 3 \mathrm{H}), 1.15-1.06(\mathrm{~m}, 3 \mathrm{H})$.

${ }^{13} \mathrm{C}$ NMR $(151 \mathrm{MHz}, \mathrm{CDCl} 3) \delta 169.14,168.08,163.98,163.40,138.07,136.92,136.32,133.48$, $132.69,132.41,129.37,128.47,128.31,127.33,127.14,126.03,112.33,106.82,101.04,74.99$, 61.70, 59.67, 55.43, 20.17, 13.89 .

ESI-HRMS: calcd for $\mathrm{C}_{26} \mathrm{H}_{26} \mathrm{~N}_{2} \mathrm{O}_{5} \mathrm{SH}^{+}\left(\left[\mathrm{M}+\mathrm{H}^{+}\right]\right)=495.1530$, Found 495.1520

(E)-ethyl 2-((3,5-dibromo-2-hydroxybenzylidene)amino)-3-(2-methylphenyl sulfonamido) -3-phenylpropanoate (7d)<smiles>CCOC(=O)C(N=Cc1cc(Br)cc(Br)c1O)C(N[S+](=O)c1ccccc1C)c1ccccc1</smiles>

$7 d$

Yellow oil; $78 \%$ yield;

${ }^{1} \mathrm{H}$ NMR $(600 \mathrm{MHz}, \mathrm{CDCl} 3) \delta 13.37(\mathrm{~s}, 1 \mathrm{H}), 8.04(\mathrm{~s}, 1 \mathrm{H})$, $7.89(\mathrm{~d}, \mathrm{~J}=8.0 \mathrm{~Hz}, 1 \mathrm{H}), 7.72(\mathrm{~d}, \mathrm{~J}=2.1 \mathrm{~Hz}, 1 \mathrm{H}), 7.33$ (t, $\mathrm{J}=7.6 \mathrm{~Hz}, 1 \mathrm{H}), 7.22(\mathrm{dd}, \mathrm{J}=9.1,6.4 \mathrm{~Hz}, 4 \mathrm{H}), 7.07(\mathrm{dd}, \mathrm{J}$ $=17.4,5.7 \mathrm{~Hz}, 3 \mathrm{H}), 5.45(\mathrm{dd}, \mathrm{J}=14.4,8.0 \mathrm{~Hz}, 1 \mathrm{H}), 4.93$ $-4.83(\mathrm{~m}, 1 \mathrm{H}), 4.48(\mathrm{~d}, \mathrm{~J}=5.7 \mathrm{~Hz}, 1 \mathrm{H}), 4.10-4.00(\mathrm{~m}$, $2 \mathrm{H}), 2.43(\mathrm{~s}, 3 \mathrm{H}), 1.11(\mathrm{t}, \mathrm{J}=7.1 \mathrm{~Hz}, 3 \mathrm{H})$.

${ }^{13} \mathrm{C}$ NMR $(151 \mathrm{MHz}, \mathrm{CDCl} 3) \delta 168.38,167.13,156.99,138.40,137.76,136.86,135.89,133.51$, $132.87,132.48,129.37,128.81,128.67,127.13,126.13,119.93,112.05,110.17,74.55,62.07$, 59.77, 20.20, 13.88 .

ESI-HRMS: calcd for $\mathrm{C}_{25} \mathrm{H}_{24} \mathrm{Br}_{2} \mathrm{~N}_{2} \mathrm{O}_{5} \mathrm{SH}^{+}\left(\left[\mathrm{M}+\mathrm{H}^{+}\right]\right)=622.9845$, Found 622.9872

E)-ethyl-((3,5-di-tert-butyl-2-hydroxybenzylidene)amino)-3-(2-methylphenylsulfonamid o)-3-phenylpropanoate (7e)<smiles>CCOC(=O)C(/N=C/c1cc(C(C)(C)C)cc(C(C)(C)C)c1O)C(NS(=O)c1ccccc1C)c1ccccc1</smiles>

$7 e$
Yellow oil; 87\% yield;

${ }^{1} \mathrm{H}$ NMR $\left(600 \mathrm{MHz}, \mathrm{CDCl}_{3}\right) \delta 12.82(\mathrm{~s}$, $1 \mathrm{H}), 12.75-12.60(\mathrm{~m}, 0.4 \mathrm{H}), 8.16(\mathrm{~s}, 1 \mathrm{H})$, $8.04(\mathrm{~s}, 0.8 \mathrm{H}), 7.90(\mathrm{~d}, J=7.9 \mathrm{~Hz}, 1 \mathrm{H}), 7.74(\mathrm{~d}$, $J=7.9 \mathrm{~Hz}, 0.8 \mathrm{H}), 7.42(\mathrm{~d}, J=2.5 \mathrm{~Hz}, 2 \mathrm{H})$, $7.28(\mathrm{~d}, J=7.6 \mathrm{~Hz}, 2 \mathrm{H}), 7.23-6.99(\mathrm{~m}, 14 \mathrm{H})$, 
$6.94(\mathrm{~d}, J=2.1 \mathrm{~Hz}, 1 \mathrm{H}), 5.64(\mathrm{~d}, J=7.8 \mathrm{~Hz}, 0.7 \mathrm{H}), 5.44(\mathrm{~d}, J=8.4 \mathrm{~Hz}, 1 \mathrm{H}), 4.98(\mathrm{dd}, J=7.7,4.9$ $\mathrm{Hz}, 0.8 \mathrm{H}), 4.93(\mathrm{dd}, J=8.1,5.5 \mathrm{~Hz}, 1 \mathrm{H}), 4.36$ (d, $J=5.3 \mathrm{~Hz}, 1 \mathrm{H}), 4.16$ (d, $J=4.8 \mathrm{~Hz}, 0.8 \mathrm{H}), 4.12$ -3.99 (m, 3H), 3.96 (dq, $J=14.4,7.2 \mathrm{~Hz}, 0.8 \mathrm{H}), 2.58$ (s, 2.5H), 2.43 (s, $3 \mathrm{H}), 1.45$ (d, $J=8.9 \mathrm{~Hz}$, $16 \mathrm{H}), 1.29(\mathrm{~d}, \mathrm{~J}=16.8 \mathrm{~Hz}, 16 \mathrm{H})$.

${ }^{13} \mathrm{C}$ NMR (151 MHz, CDCl3) $\delta 170.54,170.17,169.02,168.97,158.04,140.40,138.00$, $136.91,136.43,132.66,132.53,132.43,132.22,129.35,128.48,128.32,128.12,127.41,127.28$, $126.83,126.69,126.02,125.88,117.50,75.85,75.02,61.87,61.67,60.03,59.69,35.10,34.15$, 31.46, 31.42, 29.41, 20.28, 20.16, 13.90 .

ESI-HRMS: calcd for $\mathrm{C}_{33} \mathrm{H}_{42} \mathrm{~N}_{2} \mathrm{O}_{5} \mathrm{SH}^{+}\left(\left[\mathrm{M}+\mathrm{H}^{+}\right]\right)=579.2914$, Found 579.2905

(E)-ethyl 2-((3,5-di-tert-butyl-2-hydroxybenzylidene)amino)-3-(4-methoxy phenylsulfonamido)-3-phenylpropanoate (7f)<smiles>CCOC(N=Cc1cc(C(C)(C)C)cc(C(C)(C)C)c1O)C(N[S+](=O)c1ccc(OC)cc1)c1ccccc1</smiles>

Yellow oil; $90 \%$ yield;

${ }^{1} \mathrm{H}$ NMR $(600 \mathrm{MHz}, \mathrm{CDCl} 3) \delta 12.86(\mathrm{~s}$, $1 \mathrm{H}), 8.22(\mathrm{~s}, 1 \mathrm{H}), 7.62(\mathrm{~d}, \mathrm{~J}=8.8 \mathrm{~Hz}, 2 \mathrm{H})$, $7.43(\mathrm{~s}, 1 \mathrm{H}), 7.26(\mathrm{~s}, 1 \mathrm{H}), 7.19(\mathrm{~d}, \mathrm{~J}=2.9 \mathrm{~Hz}$, $3 \mathrm{H}), 7.11(\mathrm{~s}, 2 \mathrm{H}), 7.04(\mathrm{~d}, \mathrm{~J}=2.0 \mathrm{~Hz}, 1 \mathrm{H})$, $6.73(\mathrm{~d}, \mathrm{~J}=8.8 \mathrm{~Hz}, 2 \mathrm{H}), 5.40(\mathrm{~d}, \mathrm{~J}=8.2 \mathrm{~Hz}$, 1H), $4.94(\mathrm{dd}, \mathrm{J}=8.0,5.3 \mathrm{~Hz}, 1 \mathrm{H}), 4.37(\mathrm{~d}, \mathrm{~J}=5.2 \mathrm{~Hz}, 1 \mathrm{H}), 4.11-4.04(\mathrm{~m}, 2 \mathrm{H}), 3.75(\mathrm{~s}, 3 \mathrm{H})$, $1.45(\mathrm{~d}, \mathrm{~J}=4.1 \mathrm{~Hz}, 9 \mathrm{H}), 1.30(\mathrm{~s}, 9 \mathrm{H}), 1.12(\mathrm{t}, \mathrm{J}=7.1 \mathrm{~Hz}, 3 \mathrm{H})$.

${ }^{13} \mathrm{C}$ NMR $(151 \mathrm{MHz}, \mathrm{CDCl} 3) \delta 170.05,168.97,162.75,158.06,140.45,136.90,136.41$, $131.55,129.26,128.42,128.18,128.12,127.54,127.39,126.76,117.56,113.96,113.85,75.35$, 61.67, 59.69, 55.48, 35.10, 34.14, 31.44, 29.39, 13.93.

ESI-HRMS: calcd for $\mathrm{C}_{33} \mathrm{H}_{42} \mathrm{~N}_{2} \mathrm{O}_{6} \mathrm{SH}+\left(\left[\mathrm{M}+\mathrm{H}^{+}\right]\right)=595.2836$, Found 595.2854

(E)-ethyl2-((3,5-di-tert-butyl-2-hydroxybenzylidene)amino)-3-phenyl-3-(quinoline-8-sul fonamido)propanoate $(7 \mathrm{~g})$<smiles>CCOC(=O)C(/N=C/c1cc(C(C)(C)C)cc(C(C)(C)C)c1O)C(NS(=O)c1cccc2cccnc12)c1ccccc1</smiles>

$7 \mathrm{~g}$

Yellow oil; yield 88\%;

${ }^{1} \mathrm{HNMR}\left(600 \mathrm{MHz}, \mathrm{CDCl}_{3}\right) \delta 12.85(\mathrm{~s}, 1 \mathrm{H})$, $12.78(\mathrm{~s}, 0.7 \mathrm{H}), 9.08(\mathrm{~d}, J=2.7 \mathrm{~Hz}, 0.7 \mathrm{H}), 8.69(\mathrm{~d}$, $J=2.6 \mathrm{~Hz}, 1 \mathrm{H}), 8.20(\mathrm{~d}, J=5.0 \mathrm{~Hz}, 2 \mathrm{H}), 8.10(\mathrm{~d}, J$ $=7.6 \mathrm{~Hz}, 1.5 \mathrm{H}), 8.02(\mathrm{~d}, J=12.4 \mathrm{~Hz}, 2 \mathrm{H}), 7.81(\mathrm{~d}$, $J=8.2 \mathrm{~Hz}, 1.7 \mathrm{H}), 7.50-7.28(\mathrm{~m}, 6 \mathrm{H}), 7.08-6.97$ $(\mathrm{m}, 2 \mathrm{H}), 6.93-6.85(\mathrm{~m}, 3 \mathrm{H}), 6.82(\mathrm{dd}, J=13.7,7.5 \mathrm{~Hz}, 4.5 \mathrm{H}), 6.73(\mathrm{t}, J=7.5 \mathrm{~Hz}, 2 \mathrm{H}), 5.31$ (dd, $J=8.9,4.9 \mathrm{~Hz}, 0.7 \mathrm{H}), 5.18(\mathrm{dd}, J=9.5,5.6 \mathrm{~Hz}, 1 \mathrm{H}), 4.47(\mathrm{~d}, J=5.6 \mathrm{~Hz}, 1 \mathrm{H}), 4.15(\mathrm{~d}, J=4.9 \mathrm{~Hz}$, $0.7 \mathrm{H}), 4.11-3.87(\mathrm{~m}, 4 \mathrm{H}), 1.51(\mathrm{~s}, 7 \mathrm{H}), 1.44(\mathrm{~s}, 9 \mathrm{H}), 1.30(\mathrm{~d}, J=26.8 \mathrm{~Hz}, 17 \mathrm{H})$.

${ }^{13} \mathrm{C}$ NMR $\left(151 \mathrm{MHz}, \mathrm{CDCl}_{3}\right) \delta 170.54,170.26,168.63,158.05,157.99,151.29,150.77$, $142.99,142.80,140.32,137.42,136.92,136.76,136.54,136.45,135.29,132.84,132.54,130.05$, $129.52,128.42,128.10,127.98,127.72,127.66,127.34,126.88,126.78,125.32,121.93,121.84$, $117.49,76.42,75.61,64.42,61.67,61.51,60.56,60.17,59.55,38.16,35.12,35.08,34.14,34.10$, $31.48,31.43,31.24,29.53,29.42,25.37,13.93$.

The ee and $\mathrm{dr}$ value was determined by HPLC analysis using a Chiralpak AD-H 
$($ Hexane $/ i-\mathrm{PrOH}=96 / 4$, flow rate $1.0 \mathrm{~mL} / \mathrm{min}, \lambda=254 \mathrm{~nm}), t_{\mathrm{R}}$ (major diastereomer, $)=42.9 \mathrm{~min}$ (major enantiomer); 11.5min (minor enantiomer).

ESI-HRMS: calcd for $\mathrm{C}_{35} \mathrm{H}_{41} \mathrm{~N}_{3} \mathrm{O}_{5} \mathrm{SH}+\left(\left[\mathrm{M}+\mathrm{H}^{+}\right]\right)=616.2856$, Found 616.2864

(E)-tert-butyl 2-((3,5-di-tert-butyl-2-hydroxybenzylidene)amino)-3-phenyl-3-(quinolone-8-sulfonamido)propanoate $(7 \mathrm{~h})$<smiles>CCOC(=NC(N[S+](=O)c1cccc2cccnc12)C(OCC)C(C)(C)C)c1ccccc1</smiles>

$7 \mathrm{~h}$

Yellow oil; 87\% yield;

${ }^{1} \mathrm{H}$ NMR $\left(600 \mathrm{MHz}, \mathrm{CDCl}_{3}\right) \delta 12.99(\mathrm{~s}$, $1 \mathrm{H}), 12.91(\mathrm{~s}, 0.4 \mathrm{H}), 9.08(\mathrm{~d}, J=2.8 \mathrm{~Hz}$, $0.4 \mathrm{H}), 8.67(\mathrm{~d}, J=2.6 \mathrm{~Hz}, 1 \mathrm{H}), 8.21(\mathrm{~s}, 1 \mathrm{H})$, $8.16(\mathrm{~d}, J=7.2 \mathrm{~Hz}, 1 \mathrm{H}), 8.12-7.94(\mathrm{~m}, 2.4 \mathrm{H})$, $7.78(\mathrm{t}, J=9.4 \mathrm{~Hz}, 1.5 \mathrm{H}), 7.42(\mathrm{t}, J=7.7 \mathrm{~Hz}$, $3 \mathrm{H}), 7.38-7.28(\mathrm{~m}, 2 \mathrm{H}), 7.00(\mathrm{dd}, J=26.2$, $6.0 \mathrm{~Hz}, 2 \mathrm{H}), 6.90(\mathrm{~d}, J=2.2 \mathrm{~Hz}, 0.5 \mathrm{H}), 6.87-6.74(\mathrm{~m}, 5 \mathrm{H}), 6.70(\mathrm{dt}, J=15.0,7.6 \mathrm{~Hz}, 3 \mathrm{H}), 5.24$ $(\mathrm{dd}, J=9.4,5.0 \mathrm{~Hz}, 0.4 \mathrm{H}), 5.13(\mathrm{dd}, J=9.8,5.7 \mathrm{~Hz}, 1 \mathrm{H}), 4.38(\mathrm{~d}, J=5.7 \mathrm{~Hz}, 1 \mathrm{H}), 4.08-3.96(\mathrm{~m}$, $8 \mathrm{H}), 1.51(\mathrm{~s}, 4 \mathrm{H}), 1.45$ (s, 9H), $1.38(\mathrm{~s}, 4 \mathrm{H}), 1.32(\mathrm{~s}, 9 \mathrm{H}), 1.26(\mathrm{~d}, J=6.1 \mathrm{~Hz}, 13 \mathrm{H})$.

${ }^{13} \mathrm{C}$ NMR (151 MHz, MeOD) $\delta$ 170.16, 170.00, 167.62, 158.10, 151.19, 150.75, 142.98, $142.78,140.24,136.93,136.70,136.52,136.40,135.26,132.73,132.43,129.95,129.54,128.38$, $127.99,127.84,127.62,127.51,127.43,127.16,126.87,126.72,125.26,121.80,117.56,82.78$, $82.52,77.22,77.01,76.80,75.95,64.41,60.88,60.19,35.09,34.14,31.48,31.43,29.51,29.43$, $27.79,27.71,25.35,-0.03$.

ESI-HRMS: calcd for $\mathrm{C}_{37} \mathrm{H}_{46} \mathrm{~N}_{3} \mathrm{O}_{5} \mathrm{SH}+\left(\left[\mathrm{M}+\mathrm{H}^{+}\right]\right)=644.3159$, found 644.3171

5. Generality explorations of the Mannich-type reaction

(E)-ethyl 2-((3,5-di-tert-butyl-2-hydroxybenzylidene)amino)-3-(quinoline-8-sulfonamido)-3-(p-tolyl)propanoate<smiles>CCOC(=O)C(N=Cc1cc(C(C)(C)C)cc(C(C)(C)C)c1O)C(N[SH](=O)c1cccc2cccnc12)c1ccc(C)cc1</smiles>

7j

Yellow oil; $88 \%$ yield; $[\alpha]_{\mathrm{D}}^{20}=+0.11(\mathrm{c}=0.7$, in $\mathrm{CH}_{2} \mathrm{Cl}_{2}$;

${ }^{1} \mathrm{H}$ NMR $\left(600 \mathrm{MHz}, \mathrm{CDCl}_{3}\right) \delta 12.90(\mathrm{~s}, 1 \mathrm{H})$, $12.81(\mathrm{~s}, 0.7 \mathrm{H}), 9.06(\mathrm{dd}, J=4.2,1.6 \mathrm{~Hz}, 0.7 \mathrm{H})$, $8.68(\mathrm{dd}, J=4.2,1.6 \mathrm{~Hz}, 1 \mathrm{H}), 8.25-8.15(\mathrm{~m}$, $2.4 \mathrm{H}), 8.13-7.96(\mathrm{~m}, 3.6 \mathrm{H}), 7.88(\mathrm{~d}, J=8.1 \mathrm{~Hz}$, $0.7 \mathrm{H}), 7.82(\mathrm{~d}, J=8.0 \mathrm{~Hz}, 1.7 \mathrm{H}), 7.51-7.36(\mathrm{~m}$, $4.7 \mathrm{H}), 7.34-7.27(\mathrm{~m}, 2.5 \mathrm{H}), 7.00(\mathrm{~d}, J=2.3 \mathrm{~Hz}$, $1 \mathrm{H}), 6.92(\mathrm{dd}, J=13.2,6.0 \mathrm{~Hz}, 1.7 \mathrm{H}), 6.75(\mathrm{~d}, J=8.0 \mathrm{~Hz}, 1.4 \mathrm{H}), 6.68(\mathrm{~d}, J=8.0 \mathrm{~Hz}, 2 \mathrm{H}), 6.58(\mathrm{~d}$, $J=7.9 \mathrm{~Hz}, 1.4 \mathrm{H}), 6.48(\mathrm{~d}, J=7.8 \mathrm{~Hz}, 2 \mathrm{H}), 5.25(\mathrm{dd}, J=9.0,4.9 \mathrm{~Hz}, 0.7 \mathrm{H}), 5.13(\mathrm{dd}, J=9.6,5.7$ $\mathrm{Hz}, 1 \mathrm{H}), 4.46(\mathrm{~d}, J=5.7 \mathrm{~Hz}, 1 \mathrm{H}), 4.12(\mathrm{dd}, J=13.3,6.1 \mathrm{~Hz}, 1 \mathrm{H}), 4.05(\mathrm{qd}, J=7.1,2.9 \mathrm{~Hz}, 2 \mathrm{H})$, 3.96 (dtd, $J=17.9,10.8,7.2 \mathrm{~Hz}, 1.4 \mathrm{H}), 2.05$ (s, 2H), 2.00 (s, 3H), 1.50 (s, 6H), 1.44 (s, 9H), 1.32 (s, 9H), $1.27(\mathrm{~s}, 6 \mathrm{H}), 1.17-1.10(\mathrm{~m}, 5 \mathrm{H})$.

${ }^{13} \mathrm{C}$ NMR $\left(151 \mathrm{MHz}, \mathrm{CDCl}_{3}\right) \delta 175.14,170.48,170.24,168.70,158.06,158.00,151.25$, 
$150.75,143.00,142.84,140.28,137.41,137.25,136.86,136.74,136.45,136.29,134.59,133.94$, $132.63,132.33,131.58,130.14,129.89,129.61,128.43,128.23,128.04,127.91,127.60,127.27$, $126.88,126.79,125.60,125.31,125.27,121.97,121.90,121.79,117.51,76.49,75.62,61.64$, $61.49,60.35,59.94,35.12,35.08,34.14,34.11,31.48,31.44,29.69,29.53,29.43,21.96,21.03$, $20.78,20.73,14.20,13.98,13.94$.

The ee and $\mathrm{dr}$ value was determined by HPLC analysis using a Chiralpak AD-H $($ Hexane $/ i-\mathrm{PrOH}=90 / 10$, flow rate $1.0 \mathrm{~mL} / \mathrm{min}, \lambda=254 \mathrm{~nm}), t_{\mathrm{R}}$ (major diastereomer, $)=42.9 \mathrm{~min}$ (major enantiomer); $11.5 \mathrm{~min}$ (minor enantiomer).

ESI-HRMS: calcd for $\mathrm{C}_{36} \mathrm{H}_{43} \mathrm{~N}_{3} \mathrm{O}_{5} \mathrm{SH}^{+}\left(\left[\mathrm{M}+\mathrm{H}^{+}\right]\right)=630.2996$, Found 630.3019
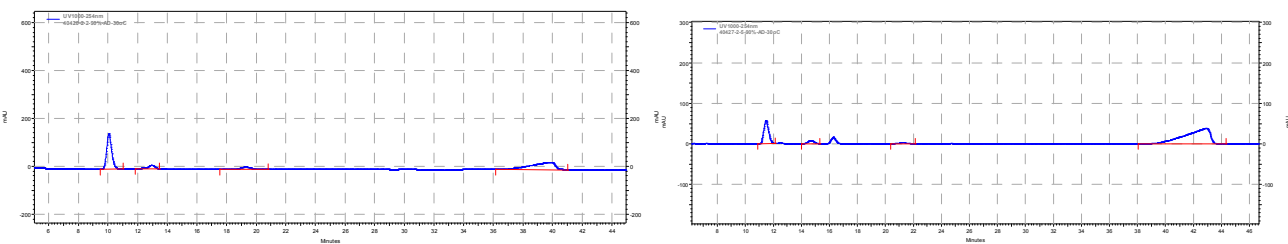

(E)-ethyl 2-((3,5-di-tert-butyl-2-hydroxybenzylidene)amino)-3-(4-methoxy-phenyl)-3(quinoline-8-sulfonamido)propanoate<smiles>CCOC(=O)C(N=Cc1cc(C(C)(C)C)cc(C(C)(C)C)c1O)C(N[SH](=O)c1cccc2cccnc12)c1ccc(OC)cc1</smiles>

$7 \mathbf{k}$

Yellow oil; $83 \%$ yield; $[\alpha]_{\mathrm{D}}^{20}=+0.26(\mathrm{c}=0.7$, in $\mathrm{CH}_{2} \mathrm{Cl}_{2}$ );

${ }^{1} \mathrm{H}$ NMR $\left(600 \mathrm{MHz}, \mathrm{CDCl}_{3}\right) \delta 12.90(\mathrm{~s}, 1 \mathrm{H}), 12.80(\mathrm{~s}$, $0.4 \mathrm{H}), 9.07(\mathrm{~s}, 0.5 \mathrm{H}), 8.69(\mathrm{~d}, J=2.7 \mathrm{~Hz}, 1 \mathrm{H}), 8.23$ $(\mathrm{s}, 1 \mathrm{H}), 8.20(\mathrm{~d}, J=7.4 \mathrm{~Hz}, 1 \mathrm{H}), 8.13-8.02(\mathrm{~m}, 2 \mathrm{H})$, $7.83(\mathrm{~d}, J=7.2 \mathrm{~Hz}, 1.6 \mathrm{H}), 7.52-7.38(\mathrm{~m}, 3.4 \mathrm{H})$, $7.32(\mathrm{dd}, J=8.2,4.2 \mathrm{~Hz}, 1 \mathrm{H}), 7.28(\mathrm{~d}, J=9.1 \mathrm{~Hz}$, $0.6 \mathrm{H}), 7.02(\mathrm{~d}, J=2.2 \mathrm{~Hz}, 1 \mathrm{H}), 6.92(\mathrm{~d}, J=9.7 \mathrm{~Hz}$, $1.4 \mathrm{H}), 6.77(\mathrm{~d}, J=8.6 \mathrm{~Hz}, 1 \mathrm{H}), 6.72(\mathrm{~d}, J=8.6 \mathrm{~Hz}, 2 \mathrm{H}), 6.30(\mathrm{~d}, J=8.7 \mathrm{~Hz}, 1 \mathrm{H}), 6.21(\mathrm{~d}, J=8.6$ $\mathrm{Hz}, 2 \mathrm{H}), 5.23(\mathrm{~s}, 0.6 \mathrm{H}), 5.13(\mathrm{dd}, J=9.5,5.6 \mathrm{~Hz}, 1 \mathrm{H}), 4.46(\mathrm{~d}, J=5.5 \mathrm{~Hz}, 1 \mathrm{H}), 4.13(\mathrm{~d}, J=4.9$ $\mathrm{Hz}, 0.5 \mathrm{H}), 4.08-3.93(\mathrm{~m}, 3 \mathrm{H}), 3.59$ (s, 1.3H), 3.57 (s, 3H), $1.51(\mathrm{~s}, 5 \mathrm{H}), 1.45(\mathrm{~s}, 9 \mathrm{H}), 1.36-1.30$ (m, 9H), 1.28 (s, 5H), $1.14(\mathrm{dt}, J=14.2,7.2 \mathrm{~Hz}, 5 \mathrm{H})$.

${ }^{13} \mathrm{C}$ NMR $\left(151 \mathrm{MHz}, \mathrm{CDCl}_{3}\right) \delta 170.49,170.33,168.69,158.77,158.05,151.26,150.77$, $142.84,140.34,136.88,136.74,136.40,132.75,130.14,129.66,128.96,128.63,128.46,128.09$, $127.98,127.53,126.91,126.80,125.36,121.84,117.52,112.95,112.65,76.60,75.68,61.67$, $61.49,60.08,59.61,55.04,35.09,34.15,31.48,31.43,29.69,29.53,29.43,13.99$.

The ee and $\mathrm{dr}$ value was determined by HPLC analysis using a Chiralpak AD-H $($ Hexane $/ i-\mathrm{PrOH}=90 / 10$, flow rate $1.0 \mathrm{~mL} / \mathrm{min}, \lambda=254 \mathrm{~nm}), t_{\mathrm{R}}$ (major diastereomer, $)=61.5 \mathrm{~min}$ (major enantiomer); 15.2min (minor enantiomer).

ESI-HRMS: calcd for $\mathrm{C}_{36} \mathrm{H}_{43} \mathrm{~N}_{3} \mathrm{O}_{6} \mathrm{SH}^{+}\left(\left[\mathrm{M}+\mathrm{H}^{+}\right]\right)=646.2945$, Found 666.2967
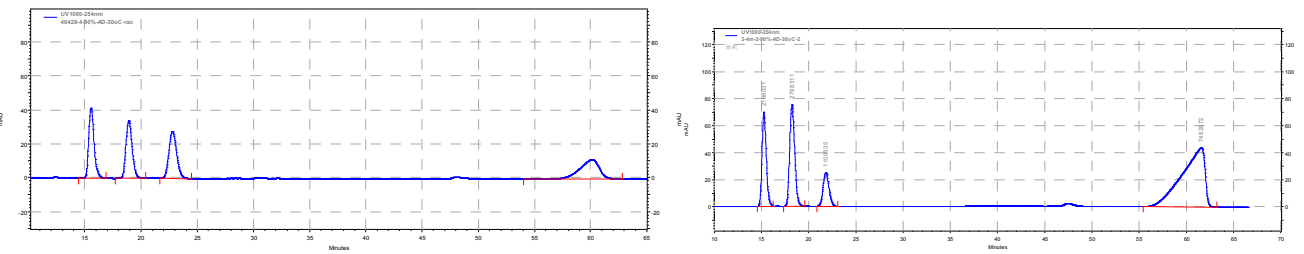
(E)-ethyl 3-(4-chlorophenyl)-2-((3,5-di-tert-butyl-2-hydroxybenzylidene)-amino)-3(quinoline-8-sulfonamido)propanoate 71<smiles>CCOC(=O)C(/N=C/c1cc(C(C)(C)C)cc(C(C)(C)C)c1O)C(NS(=O)c1cccc2cccnc12)c1ccc(Cl)cc1</smiles>

71

Yellow oil; $91 \%$ yield; $[\alpha]_{\mathrm{D}}^{20}=+0.19(\mathrm{c}=1.2$, in $\mathrm{CH}_{2} \mathrm{Cl}_{2}$ );

${ }^{1} \mathrm{H}$ NMR $\left(600 \mathrm{MHz}, \mathrm{CDCl}_{3}\right) \delta 12.76(\mathrm{~s}, 1 \mathrm{H})$, $12.66(\mathrm{~s}, 0.3 \mathrm{H}), 9.08(\mathrm{~s}, 0.3 \mathrm{H}), 8.68(\mathrm{~d}, J=2.7 \mathrm{~Hz}$, $1 \mathrm{H}), 8.26(\mathrm{~s}, 1 \mathrm{H}), 8.18(\mathrm{~d}, J=7.2 \mathrm{~Hz}, 1 \mathrm{H}), 8.15(\mathrm{~d}$, $J=7.1 \mathrm{~Hz}, 0.3 \mathrm{H}), 8.08(\mathrm{dd}, J=19.3,11.0 \mathrm{~Hz}$, $1.5 \mathrm{H}), 7.88(\mathrm{~d}, J=8.1 \mathrm{~Hz}, 1.3 \mathrm{H}), 7.47(\mathrm{dd}, J=16.9$, $9.1 \mathrm{~Hz}, 2.9 \mathrm{H}), 7.41-7.33(\mathrm{~m}, 1.4 \mathrm{H}), 7.04(\mathrm{~d}, J=$ $2.2 \mathrm{~Hz}, 1.3 \mathrm{H}), 6.98-6.91(\mathrm{~m}, 1.3 \mathrm{H}), 6.84(\mathrm{~d}, J=8.5 \mathrm{~Hz}, 0.6 \mathrm{H}), 6.79-6.71(\mathrm{~m}, 2.5 \mathrm{H}), 6.65(\mathrm{~d}, J$ $=8.4 \mathrm{~Hz}, 2 \mathrm{H}), 5.17(\mathrm{dd}, J=9.6,5.5 \mathrm{~Hz}, 1 \mathrm{H}), 4.46(\mathrm{~d}, J=5.5 \mathrm{~Hz}, 1 \mathrm{H}), 4.12(\mathrm{~d}, J=4.8 \mathrm{~Hz}, 0.3 \mathrm{H})$, $4.09-4.00$ (m, 2H), $4.00-3.92$ (m, 0.6H), 1.50 (s, 3H), 1.44 (d, $J=9.5 \mathrm{~Hz}, 9 \mathrm{H}), 1.33$ (s, 9H), $1.28(\mathrm{~s}, 3 \mathrm{H}), 1.13(\mathrm{dd}, J=15.1,8.0 \mathrm{~Hz}, 4 \mathrm{H})$.

${ }^{13} \mathrm{C}$ NMR $\left(151 \mathrm{MHz}, \mathrm{CDCl}_{3}\right) \delta 170.73,168.37,158.00,150.84,142.72,140.56,136.84$, $136.67,136.62,133.92,133.60,132.93,130.21,129.66,129.17,128.79,128.47,128.25,127.76$, $127.33,126.99,125.41,122.01,117.43,75.37,61.85,61.67,59.39,53.40,35.10,34.17,31.47$, $31.42,29.69,29.51,29.42,13.96$.

The ee and dr value was determined by HPLC analysis using a Chiralpak AD-H (Hexane $/ i$-PrOH $=95 / 5-80 / 20$, flow rate $1.0 \mathrm{~mL} / \mathrm{min}, \lambda=254 \mathrm{~nm}), t_{\mathrm{R}}$ (major diastereomer, $)=$ $60.9 \mathrm{~min}$ (major enantiomer); $27.1 \mathrm{~min}$ (minor enantiomer).

ESI-HRMS: calcd for $\mathrm{C}_{36} \mathrm{H}_{43} \mathrm{~N}_{3} \mathrm{O}_{6} \mathrm{SH}^{+}\left(\left[\mathrm{M}+\mathrm{H}^{+}\right]\right)=649.2394$, Found 649.2381
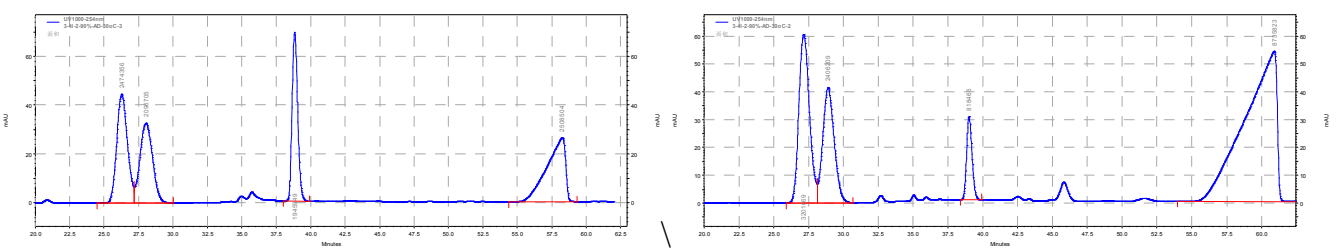

(E)-ethyl 2-((3,5-di-tert-butyl-2-hydroxybenzylidene)amino)-3-(quinoline-8-sulfonamido)-3-(o-tolyl)propanoate<smiles>CCOC(=N/C=C/c1cc(C(C)(C)C)cc(C(C)(C)C)c1O)C(N[S@](=O)c1cccc2cccnc12)c1ccccc1C</smiles>

$7 m$

Yellow oil; $87 \%$ yield; $[\alpha]_{\mathrm{D}}^{20}=+0.25(\mathrm{c}=1.2$, in $\mathrm{CH}_{2} \mathrm{Cl}_{2}$;

${ }^{1} \mathrm{H}$ NMR $\left(600 \mathrm{MHz}, \mathrm{CDCl}_{3}\right) \delta 12.87(\mathrm{~s}, 1 \mathrm{H}), 8.79$ $-8.76(\mathrm{~m}, 1 \mathrm{H}), 8.23(\mathrm{~d}, J=7.3 \mathrm{~Hz}, 1 \mathrm{H}), 8.05(\mathrm{~s}, 1 \mathrm{H})$, $8.01-7.98(\mathrm{~m}, 1 \mathrm{H}), 7.79(\mathrm{~d}, J=8.1 \mathrm{~Hz}, 1 \mathrm{H}), 7.43$ (dd, $J=13.4,5.5 \mathrm{~Hz}, 1 \mathrm{H}), 7.37(\mathrm{~d}, J=2.1 \mathrm{~Hz}, 1 \mathrm{H})$, $7.32(\mathrm{dd}, J=8.3,4.2 \mathrm{~Hz}, 1 \mathrm{H}), 6.96(\mathrm{~d}, J=9.7 \mathrm{~Hz}$, $1 \mathrm{H}), 6.87(\mathrm{dd}, J=15.7,5.0 \mathrm{~Hz}, 2 \mathrm{H}), 6.65-6.53(\mathrm{~m}, 2 \mathrm{H}), 6.43(\mathrm{t}, J=7.4 \mathrm{~Hz}, 1 \mathrm{H}), 5.49$ (dd, $J=$ 9.6, 7.4 Hz, 1H), 4.39 (d, J=7.3 Hz, 1H), $4.24-4.03$ (m, 2H), 2.09 (s, 3H), 1.41 (s, 9H), 1.27 (s, $9 \mathrm{H}), 1.18(\mathrm{t}, J=7.1 \mathrm{~Hz}, 3 \mathrm{H})$.

${ }^{13} \mathrm{C}$ NMR $\left(151 \mathrm{MHz}, \mathrm{CDCl}_{3}\right) \delta 169.58,168.99,157.98,151.17,150.74,142.74,140.20$, $136.80,136.73,136.42,136.07,134.65,134.32,132.90,132.76,131.38,129.76,129.69,128.35$, $127.90,127.32,126.70,126.23,125.20,124.98,121.82,117.38,77.22,77.01,76.80,76.29,61.63$, 
$55.19,35.04,34.09,31.43,29.36,19.25,13.94$.

The ee and dr value was determined by HPLC analysis using a Chiralpak AD-H $($ Hexane $/ i-\mathrm{PrOH}=90 / 10$, flow rate $1.0 \mathrm{~mL} / \mathrm{min}, \lambda=254 \mathrm{~nm}), t_{\mathrm{R}}$ (major diastereomer, $)=42.9 \mathrm{~min}$ (major enantiomer); 11.5min (minor enantiomer).

ESI-HRMS: calcd for $\mathrm{C}_{36} \mathrm{H}_{43} \mathrm{~N}_{3} \mathrm{O}_{5} \mathrm{SH}^{+}\left(\left[\mathrm{M}+\mathrm{H}^{+}\right]\right)=630.2996$, Found 630.3021
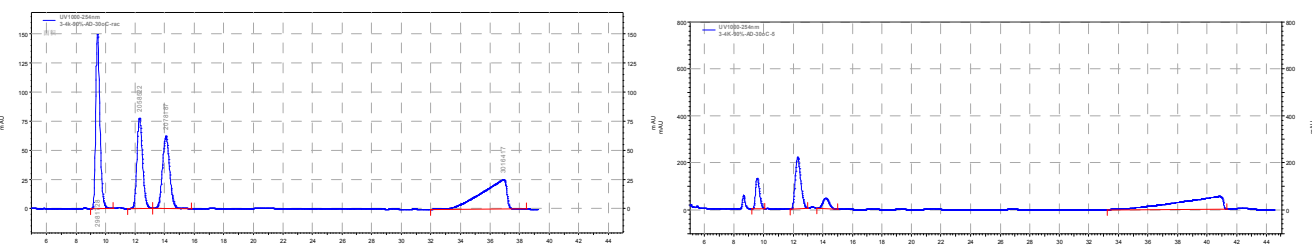

(E)-ethyl 2-((3,5-di-tert-butyl-2-hydroxybenzylidene)amino)-3-(quinoline-8-sulfonamido)-3-(thiophen-2-yl)propanoate<smiles>CCOC(=N/C=C/c1cc(C(C)(C)C)cc(C(C)(C)C)c1O)C(NS(=O)c1cccc2cccnc12)c1cccs1</smiles>

$7 n$

Yellow oil; $90 \%$ yield; $[\alpha]_{\mathrm{D}}^{20}=+0.13$ ( $\mathrm{c}=1.0$, in $\mathrm{CH}_{2} \mathrm{Cl}_{2}$ );

${ }^{1} \mathrm{H}$ NMR $\left(600 \mathrm{MHz}, \mathrm{CDCl}_{3}\right) \delta 12.67(\mathrm{~s}$, $0.6 \mathrm{H}), 12.60(\mathrm{~s}, 1 \mathrm{H}), 9.15(\mathrm{~d}, J=2.6 \mathrm{~Hz}, 0.6 \mathrm{H})$, $8.71-8.58(\mathrm{~m}, 1 \mathrm{H}), 8.29(\mathrm{~s}, 1 \mathrm{H}), 8.22(\mathrm{~d}, J=$ $7.2 \mathrm{~Hz}, 1 \mathrm{H}), 8.17(\mathrm{~s}, 0.6 \mathrm{H}), 8.11(\mathrm{dd}, J=15.0$, $7.7 \mathrm{~Hz}, 1.2 \mathrm{H}), 8.05(\mathrm{~d}, J=8.3 \mathrm{~Hz}, 1 \mathrm{H}), 7.86(\mathrm{~d}, J=8.2 \mathrm{~Hz}, 1 \mathrm{H}), 7.82(\mathrm{~d}, J=8.2 \mathrm{~Hz}, 0.6 \mathrm{H}), 7.57-$ $7.40(\mathrm{~m}, 4.6 \mathrm{H}), 7.30(\mathrm{dd}, J=8.3,4.3 \mathrm{~Hz}, 1 \mathrm{H}), 7.11-6.95(\mathrm{~m}, 2.7 \mathrm{H}), 6.69(\mathrm{~d}, J=5.0 \mathrm{~Hz}, 1 \mathrm{H})$, $6.63(\mathrm{~d}, J=5.0 \mathrm{~Hz}, 0.6 \mathrm{H}), 6.54(\mathrm{~d}, J=3.4 \mathrm{~Hz}, 0.6 \mathrm{H}), 6.49(\mathrm{~d}, J=3.4 \mathrm{~Hz}, 1 \mathrm{H}), 6.41-6.26(\mathrm{~m}$, 1.6H), $5.74-5.65(\mathrm{~m}, 0.6 \mathrm{H}), 5.58(\mathrm{dd}, J=9.8,4.6 \mathrm{~Hz}, 1 \mathrm{H}), 4.49$ (d, $J=4.6 \mathrm{~Hz}, 1 \mathrm{H}), 4.27-4.16$ (m, 1H), 4.12 (q, $J=7.1 \mathrm{~Hz}, 1.6 \mathrm{H}), 4.03(\mathrm{dt}, J=7.1,4.5 \mathrm{~Hz}, 2 \mathrm{H}), 1.53(\mathrm{~s}, 5 \mathrm{H}), 1.49$ (s, 9H), 1.34 (s, 9H), $1.28(\mathrm{~s}, 5 \mathrm{H}), 1.08(\mathrm{t}, J=7.1 \mathrm{~Hz}, 3 \mathrm{H})$.

${ }^{13} \mathrm{C}$ NMR $\left(151 \mathrm{MHz}, \mathrm{CDCl}_{3}\right) \delta 171.82,171.56,168.44,168.16,157.99,157.90,151.54$, $151.02,142.79,140.49,138.98,137.23,136.86,136.71,136.48,136.41,132.84,132.51,129.87$, $129.47,128.39,128.31,128.23,127.79,127.29,127.00,125.64,125.42,125.27,125.20,125.15$, $121.91,121.87,117.54,75.85,75.42,62.05,61.59,60.38,56.14,55.74,35.14,34.18,34.13,31.49$, $31.42,29.69,29.54,29.50,14.19,14.11,13.90$.

The ee and dr value was determined by HPLC analysis using a Chiralpak AD-H $\left(\right.$ Hexane $/ i-\mathrm{PrOH}=90 / 10$, flow rate $1.0 \mathrm{~mL} / \mathrm{min}, \lambda=254 \mathrm{~nm}$ ), $t_{\mathrm{R}}$ (major diastereomer, $)=36.8 \mathrm{~min}$ (major enantiomer); $12.0 \mathrm{~min}$ (minor enantiomer).

ESI-HRMS: calcd for $\mathrm{C}_{33} \mathrm{H}_{39} \mathrm{~N}_{3} \mathrm{O}_{5} \mathrm{~S}_{2} \mathrm{H}^{+}\left(\left[\mathrm{M}+\mathrm{H}^{+}\right]\right)=622.2444$, Found 622.2433
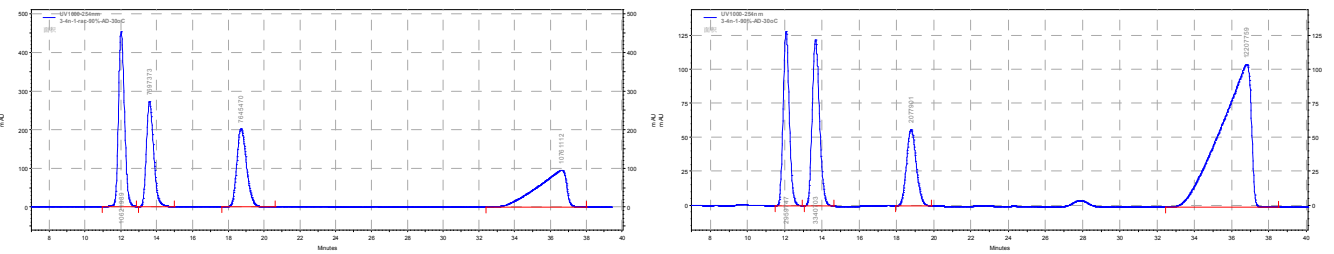

(E)-ethyl 2-((3,5-di-tert-butyl-2-hydroxybenzylidene)amino)-3-(naphthalen $\quad$-1-yl)-3- 
(quinoline-8-sulfonamido)propanoate (70)<smiles>CCOC(=O)C(/N=C/c1cc(C(C)(C)C)cc(C(C)(C)C)c1O)C(N[SH](=O)(OC)c1cccc2cccnc12)c1cccc2ccccc12</smiles>

Yellow oil; $82 \%$ yield; $[\alpha]_{\mathrm{D}}^{20}=+0.17 \quad(\mathrm{c}=0.9$, in $\mathrm{CH}_{2} \mathrm{Cl}_{2}$;

${ }^{1} \mathrm{H}$ NMR $\left(600 \mathrm{MHz}, \mathrm{CDCl}_{3}\right) \delta 12.83(\mathrm{~s}, 1 \mathrm{H}), 8.59(\mathrm{~s}$, $1 \mathrm{H}), 8.14(\mathrm{~s}, 1 \mathrm{H}), 8.05(\mathrm{~d}, J=7.3 \mathrm{~Hz}, 1 \mathrm{H}), 7.97(\mathrm{~d}, J=$ $8.5 \mathrm{~Hz}, 1 \mathrm{H}), 7.83(\mathrm{~d}, J=8.2 \mathrm{~Hz}, 1 \mathrm{H}), 7.58(\mathrm{~d}, J=8.1 \mathrm{~Hz}$, $1 \mathrm{H}), 7.50(\mathrm{~d}, J=8.1 \mathrm{~Hz}, 1 \mathrm{H}), 7.35(\mathrm{dd}, J=10.0,5.2 \mathrm{~Hz}$, 2H), $7.29(\mathrm{t}, J=7.7 \mathrm{~Hz}, 2 \mathrm{H}), 7.21(\mathrm{~s}, 1 \mathrm{H}), 7.19-7.13(\mathrm{~m}$, 2H), 7.10 (d, $J=9.5 \mathrm{~Hz}, 1 \mathrm{H}), 6.89$ (s, 1H), 6.81 (t, $J=7.7 \mathrm{~Hz}, 1 \mathrm{H}), 6.04$ (s, 1H), 4.68 (d, $J=6.5$ $\mathrm{Hz}, 1 \mathrm{H}), 4.08-3.83$ (m, 2H), 1.39 (s, 9H), 1.28 (s, 9H), 1.03 (d, $J=6.6 \mathrm{~Hz}, 3 \mathrm{H})$.

${ }^{13} \mathrm{C}$ NMR $\left(151 \mathrm{MHz}, \mathrm{CDCl}_{3}\right) \delta 169.80,168.87,157.98,150.50,142.46,140.17,136.68$, $136.30,136.09,133.05,132.68,130.16,128.29,128.24,128.04,127.89,126.76,126.21,125.28$, $124.93,124.11,122.84,121.56,117.36,75.63,61.59,35.02,34.09,31.44,29.69,29.36,13.80$.

The ee and $\mathrm{dr}$ value was determined by HPLC analysis using a Chiralpak AD-H $\left(\right.$ Hexane $/ i-\mathrm{PrOH}=90 / 10$, flow rate $1.0 \mathrm{~mL} / \mathrm{min}, \lambda=254 \mathrm{~nm}$ ), $t_{\mathrm{R}}$ (major diastereomer, $)=37.8 \mathrm{~min}$ (major enantiomer); $11.1 \mathrm{~min}$ (minor enantiomer).

ESI-HRMS: calcd for $\mathrm{C}_{39} \mathrm{H}_{43} \mathrm{~N}_{3} \mathrm{O}_{5} \mathrm{SH}^{+}\left(\left[\mathrm{M}+\mathrm{H}^{+}\right]\right)=666.2996$, Found 666.3014

6. Determination of absolute configuration for Mannich main adducts<smiles>CC(=O)CN=Cc1cc([18F])cc([18OH])c1O</smiles>

$4 g$ 5a $7 p$

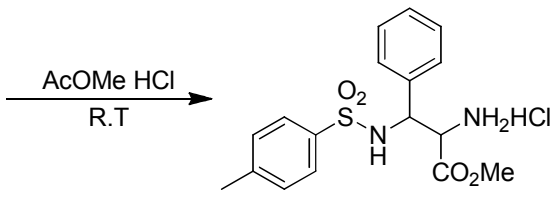

$8 a$
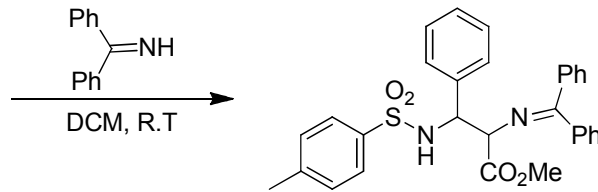

$9 a$

Scheme S10. Determination of absolute configuration

According to the above procedure, afford $7 \mathbf{p}$, light yellow oil; $80 \%$ yield; Saturated $\mathrm{HCl}$ solution of $\mathrm{AcOMe}(6 \mathrm{ml})$ was added slowly to a stirred solution of $7 \mathbf{p}(1 \mathrm{~g}, 1.5 \mathrm{mmol})$ in $\mathrm{CH}_{2} \mathrm{Cl}_{2}(10 \mathrm{ml})$ at $0 \mathrm{oC}$. The solution was stirred for $2 \mathrm{~h}$ at $0^{\circ} \mathrm{C}$ and warmed to room temperature and was stirred for another $5 \mathrm{~h}$. After that, the obtained solution was dried and evaporated to afford crude product $8 \mathrm{a}$ as yellow solid. Crude solid $8 \mathrm{a}(0.40 \mathrm{~g})$, benzophenone imine and $10 \mathrm{ml} \mathrm{CH}_{2} \mathrm{Cl}_{2}$, were stirred at room temperature for $20 \mathrm{~h}$ with the exclusion of moisture $\left(\mathrm{CaCl}_{2}\right.$ tube). The reaction mixture was filtered to remove $\mathrm{NH}_{4} \mathrm{Cl}$ and evaporated. The residue was purified by flash chromatography on silica gel to afford 9a (140 mg, 42\% yield in two steps) as white solid, matching the previously published characterization data ${ }^{[9]}$.<smiles>COC(=O)C(/N=C/c1cc(C(C)(C)C)cc(C(C)(C)C)c1O)C(NS(=O)(=O)c1ccc(C)cc1)c1ccccc1</smiles>

7p, Yellow oil, $80 \%$ yield, $\left.1 \mathrm{H} \mathrm{NMR} \mathrm{(600} \mathrm{MHz,} \mathrm{CDCl}_{3}\right)$ $\delta 12.78(\mathrm{~s}, 1 \mathrm{H}), 12.59(\mathrm{~s}, 1 \mathrm{H}), 8.20(\mathrm{~s}, 1 \mathrm{H}), 8.00(\mathrm{~s}$, 
1H), $7.57(\mathrm{t}, \mathrm{J}=9.1 \mathrm{~Hz}, 3 \mathrm{H}), 7.43(\mathrm{~s}, 2 \mathrm{H}), 7.22-7.14(\mathrm{~m}, 5 \mathrm{H}), 7.09$ (d, $J=7.3 \mathrm{~Hz}, 7 \mathrm{H}), 7.03(\mathrm{~d}, J$ $=2.1 \mathrm{~Hz}, 1 \mathrm{H}), 6.92(\mathrm{~d}, J=2.1 \mathrm{~Hz}, 1 \mathrm{H}), 5.64(\mathrm{~d}, J=7.6 \mathrm{~Hz}, 1 \mathrm{H}), 5.37(\mathrm{~s}, 1 \mathrm{H}), 5.03(\mathrm{~s}, 1 \mathrm{H}), 5.01-$ $4.92(\mathrm{~m}, 1 \mathrm{H}), 4.40(\mathrm{~d}, J=5.2 \mathrm{~Hz}, 1 \mathrm{H}), 4.20(\mathrm{~d}, J=4.7 \mathrm{~Hz}, 1 \mathrm{H}), 3.61(\mathrm{~s}, 3 \mathrm{H}), 3.59$ (s, 2H), $2.32(\mathrm{~s}$, 2H), $2.30(\mathrm{~s}, 3 \mathrm{H}), 1.45(\mathrm{~s}, 6 \mathrm{H}), 1.44(\mathrm{~s}, 9 \mathrm{H}), 1.30(\mathrm{~s}, 10 \mathrm{H}), 1.29-1.24(\mathrm{~m}, 7 \mathrm{H})$.

${ }^{13} \mathrm{C}$ NMR $\left(151 \mathrm{MHz}, \mathrm{CDCl}_{3}\right) \delta 170.74,170.29,169.39,158.03,157.90,143.34,143.16,140.48$, $137.39,136.96,136.34,129.45,129.33,128.45,128.39,128.20,127.93,127.46,127.26,127.12$, $126.78,126.71,123.07,117.49,75.99,75.42,59.90,59.66,52.60,52.45,35.09,34.14,31.45$, 31.40, 29.43, 29.40, 21.43.

ESI-HRMS: calcd for $\mathrm{C}_{32} \mathrm{H}_{40} \mathrm{~N}_{2} \mathrm{O}_{5} \mathrm{SH}^{+}\left(\left[\mathrm{M}+\mathrm{H}^{+}\right]\right)=565.2731$, Found 565.2714

The ee and $\mathrm{dr}$ value was determined by HPLC analysis using a Chiralpak AD-H $\left(\right.$ Hexane $/ i-\mathrm{PrOH}=90 / 10$, flow rate $1.0 \mathrm{~mL} / \mathrm{min}, \lambda=254 \mathrm{~nm}$ ), $t_{\mathrm{R}}$ (major diastereomer, $)=33.8 \mathrm{~min}$ (major enantiomer); $37.0 \mathrm{~min}$ (minor enantiomer). major diastereomer $(\mathrm{ee}=29$ )/ minor diastereomer $(\mathrm{ee}=42)=1.7: 1$,
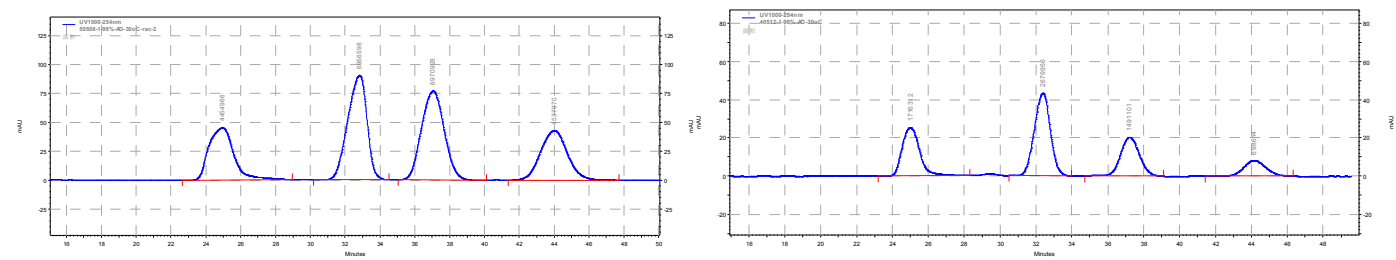

Product 9a Match the previously published characterization data. The ee and dr value was determined by HPLC analysis using a Chiralpak AD-H (Hexane/ i-PrOH $=93 / 7$, flow rate 1 $\mathrm{mL} / \mathrm{min}, \lambda=254 \mathrm{~nm}), t_{\mathrm{R}}=21.5 \mathrm{~min}(\mathbf{2} \boldsymbol{R}, 3 \boldsymbol{S})$-isomer, $35.2 \mathrm{~min}(\mathbf{R}, 3 \boldsymbol{R})$-isomer, $43.7 \mathrm{~min}$ (2S,3S)-isomer, 48.0 min (2S,3R)-isomer, ESI-HRMS: calcd for $\mathrm{C}_{30} \mathrm{H}_{28} \mathrm{~N}_{2} \mathrm{O}_{5} \mathrm{SH}^{+}\left(\left[\mathrm{M}+\mathrm{H}^{+}\right]\right)=$ 513.1843, Found 513.1868 and $\mathrm{C}_{30} \mathrm{H}_{28} \mathrm{~N}_{2} \mathrm{O}_{5} \mathrm{SNa}^{+}\left(\left[\mathrm{M}+\mathrm{Na}^{+}\right]\right)=535.1662$, Found 535.1684;

Major diastereomer $(\mathrm{ee}=26) /$ minor diastereomer $(\mathrm{ee}=43)=1.1: 1$. Therefore, the ee of major diastereomer is in accord with 9a. Then, according to the literature of Shin-ichi Fukuzawa and co-workers ${ }^{[9]}$, compared $(2 S, 3 S)$-methyl 2-((diphenylmethylene)amino)-3-(4-methylphenylsulfonamido)-3-phenylpropanoate with other isomer, the absolute configuration of this mainly Mannich product is $(2 S, 3 S)$.

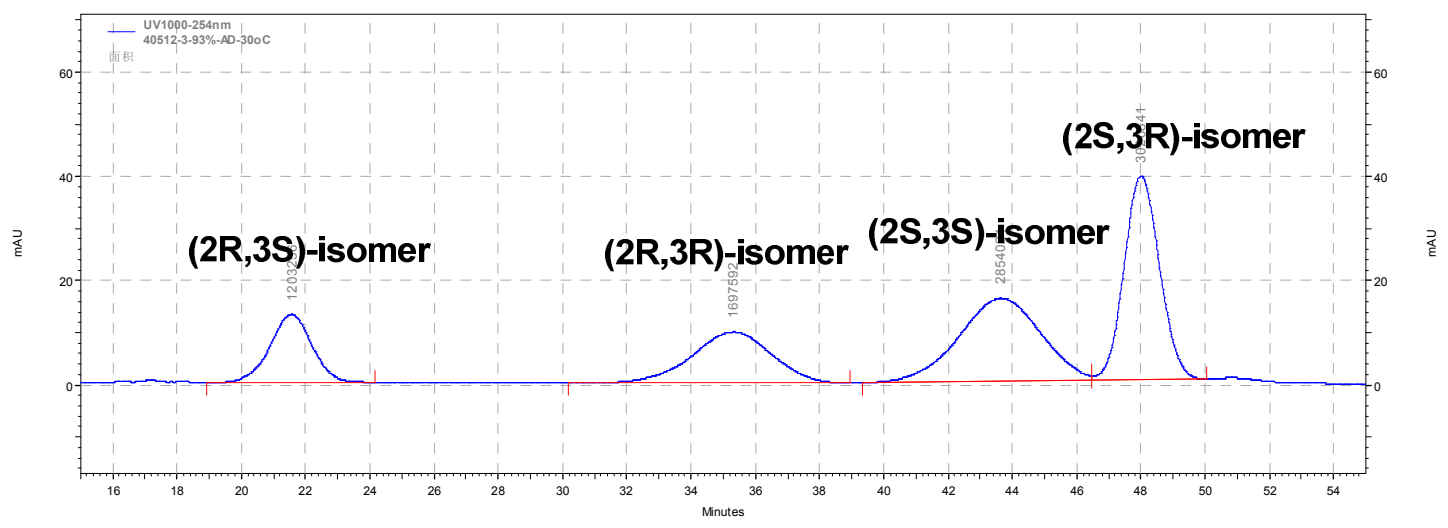


7. NMR spectra

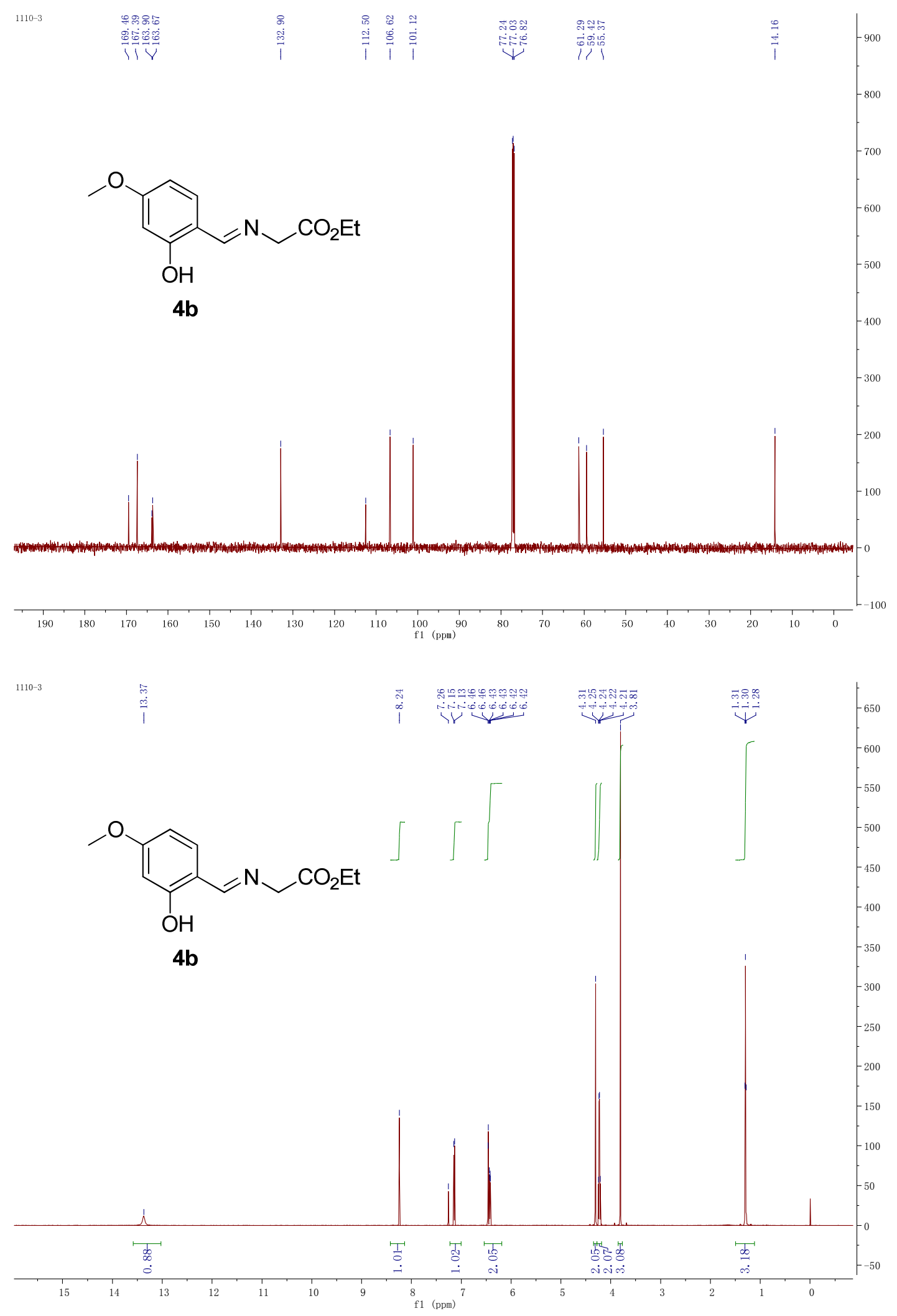




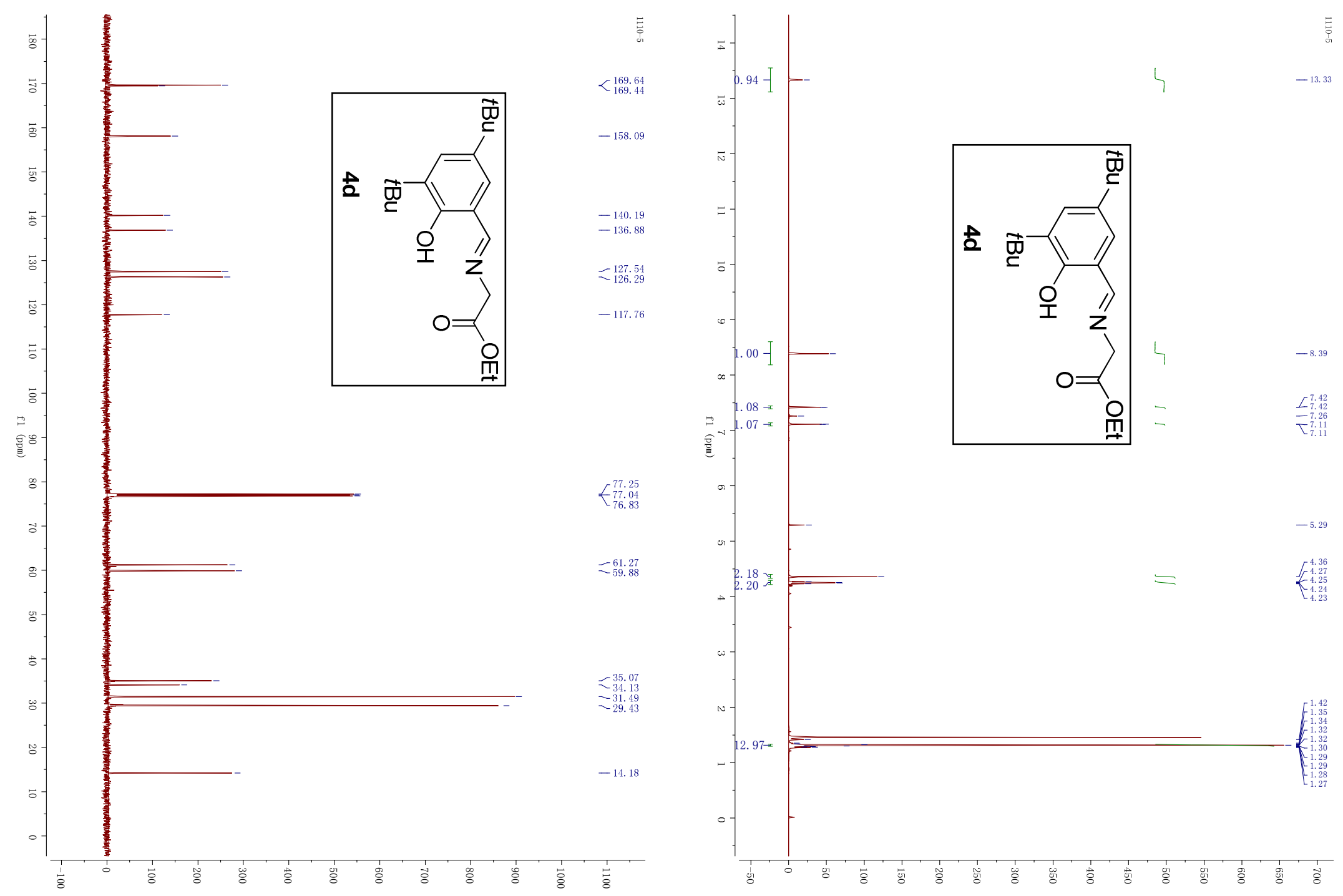



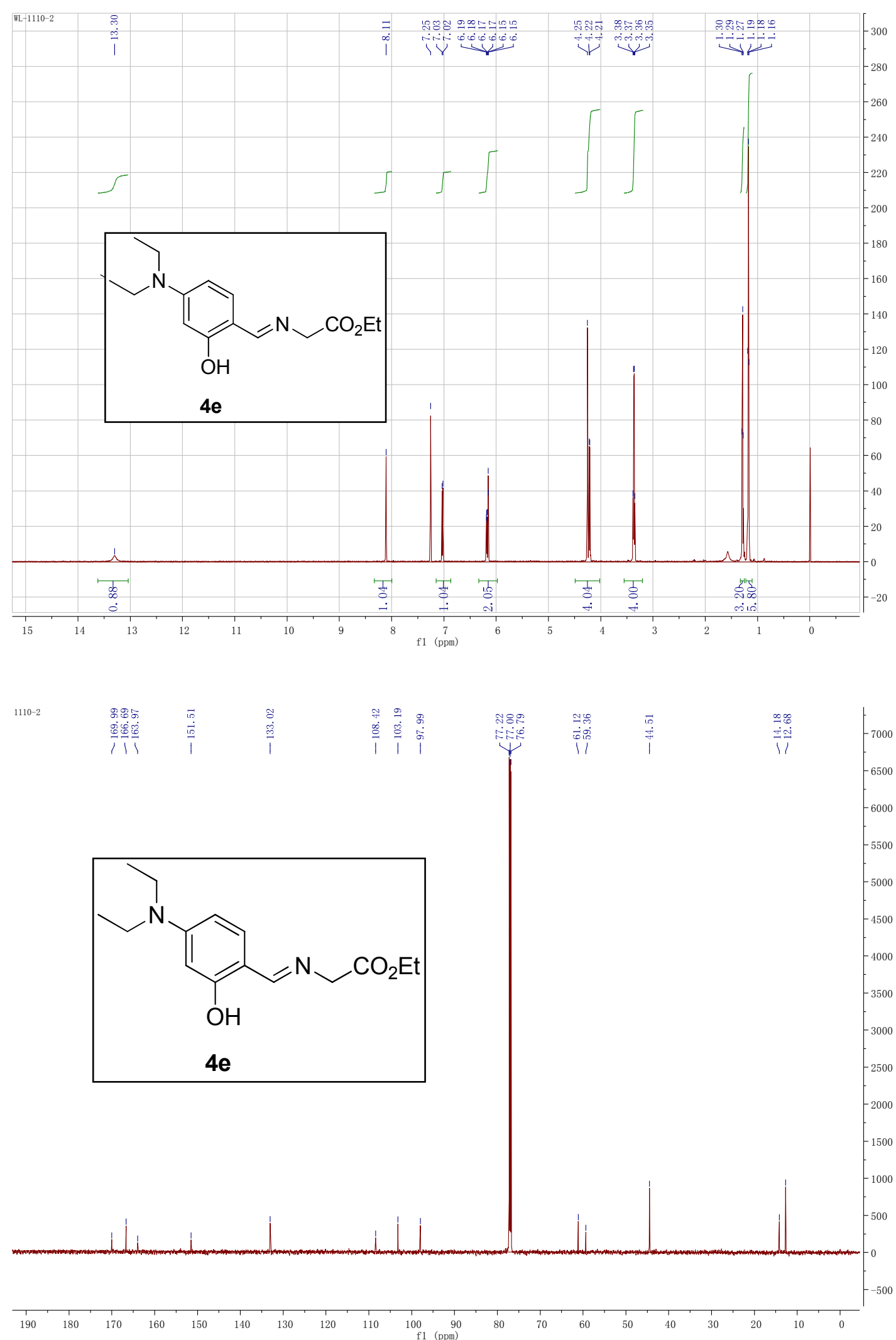

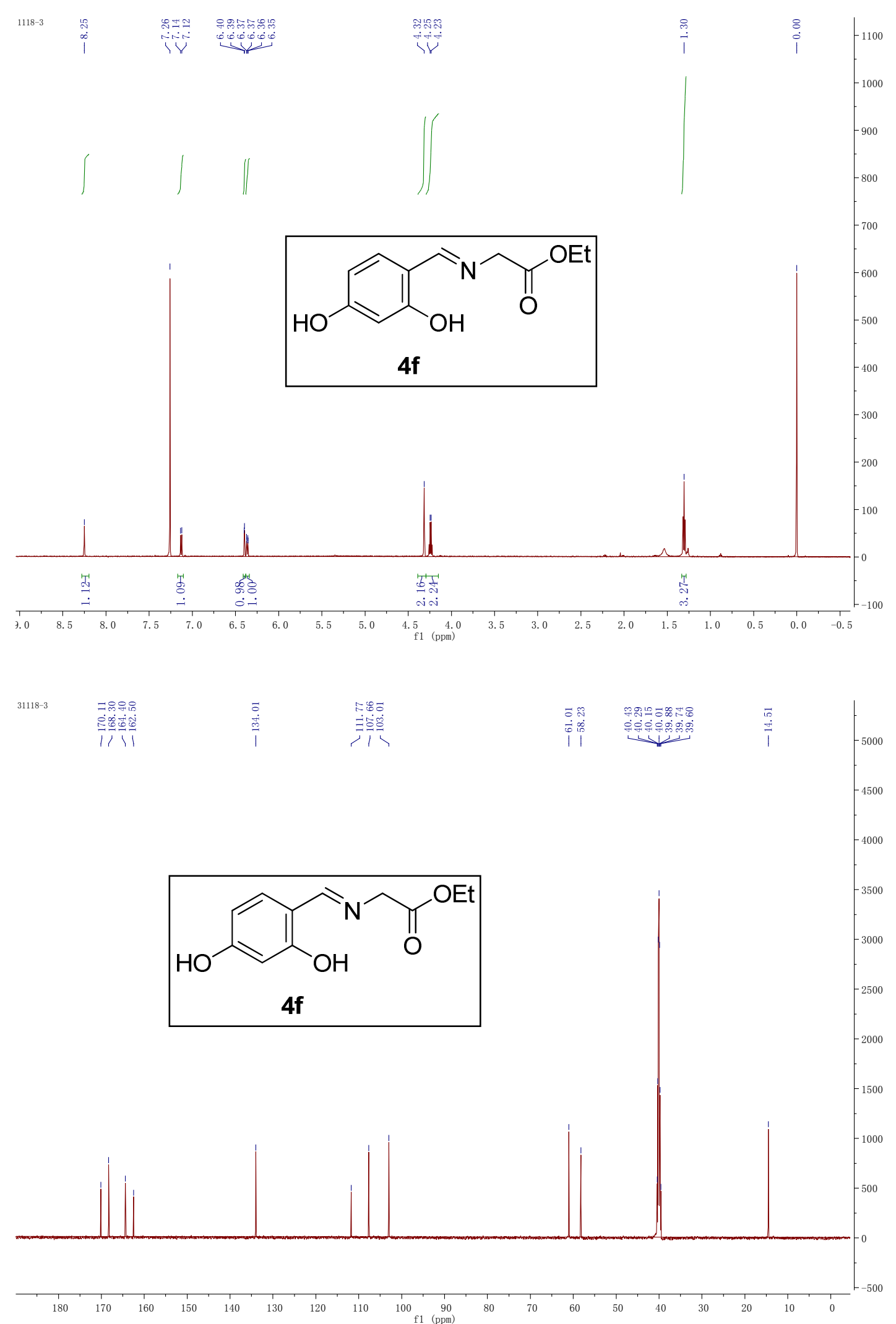


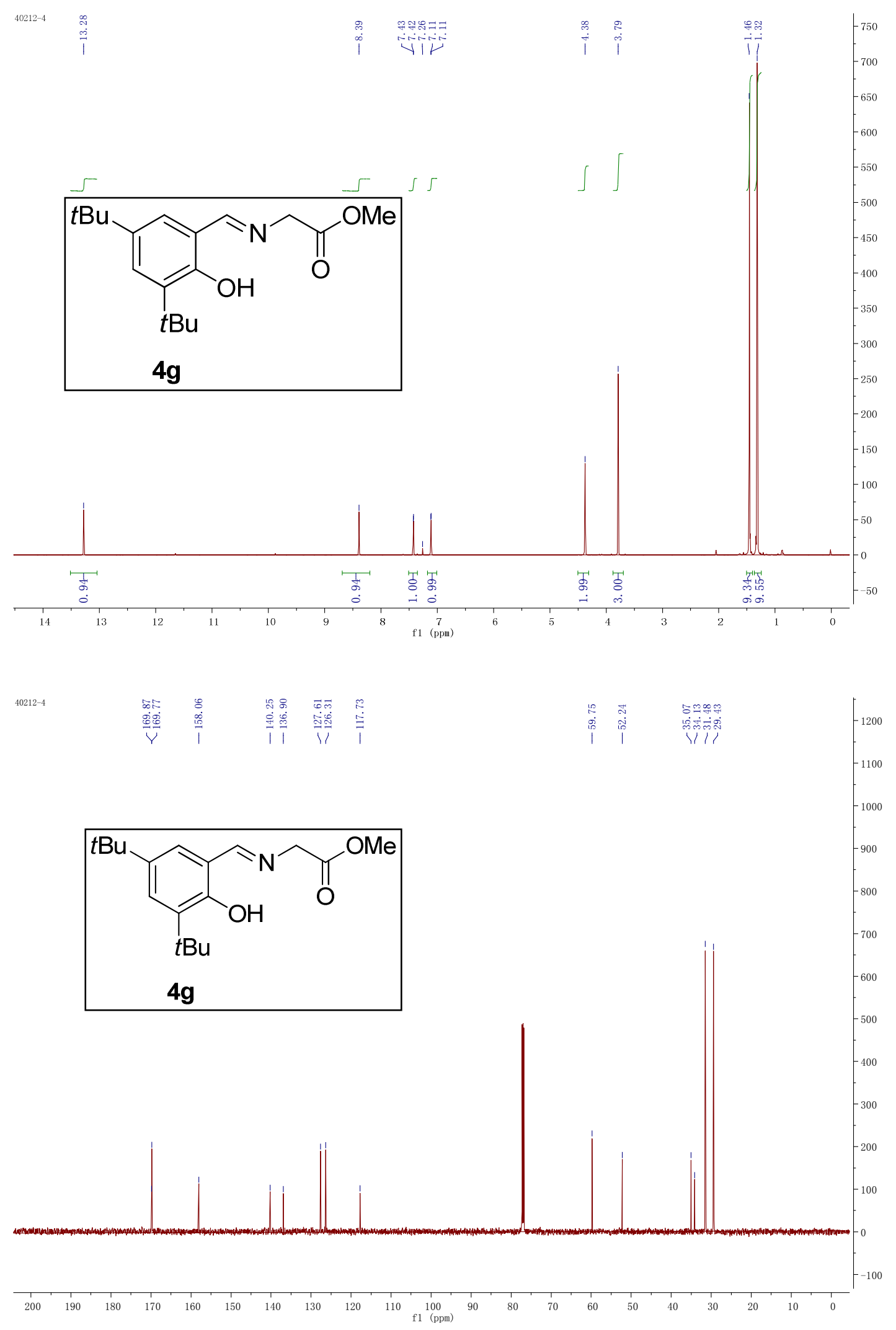




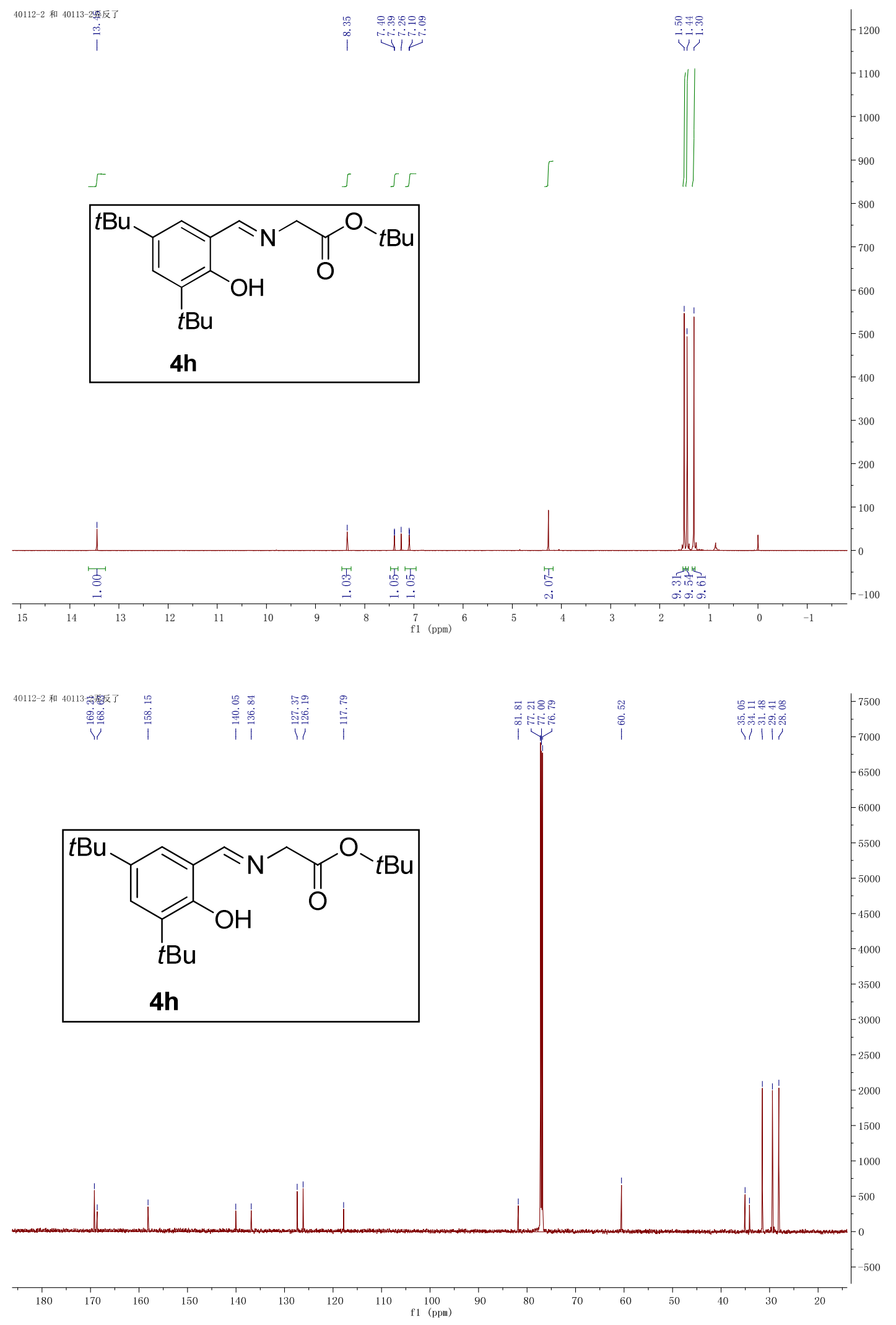



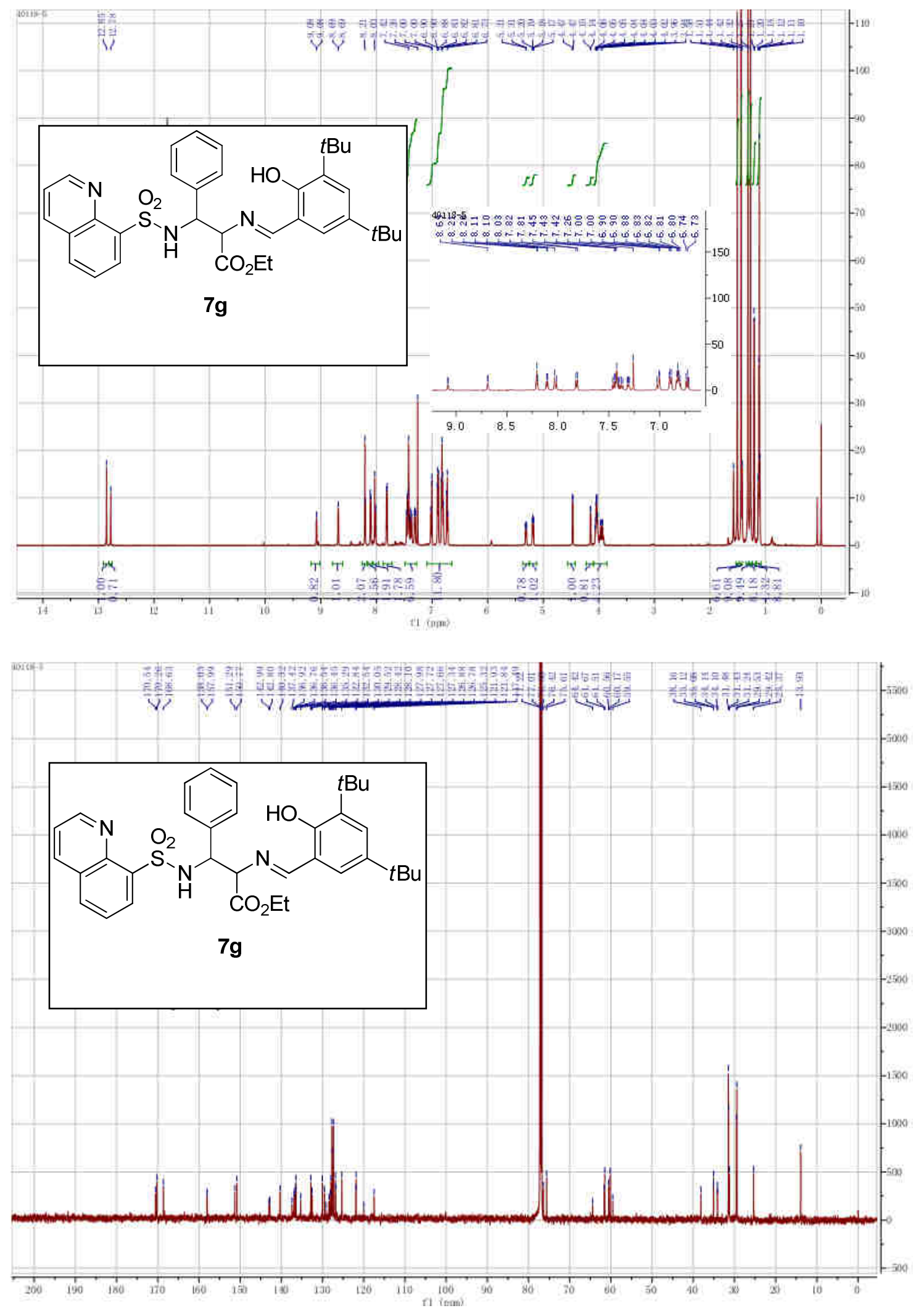

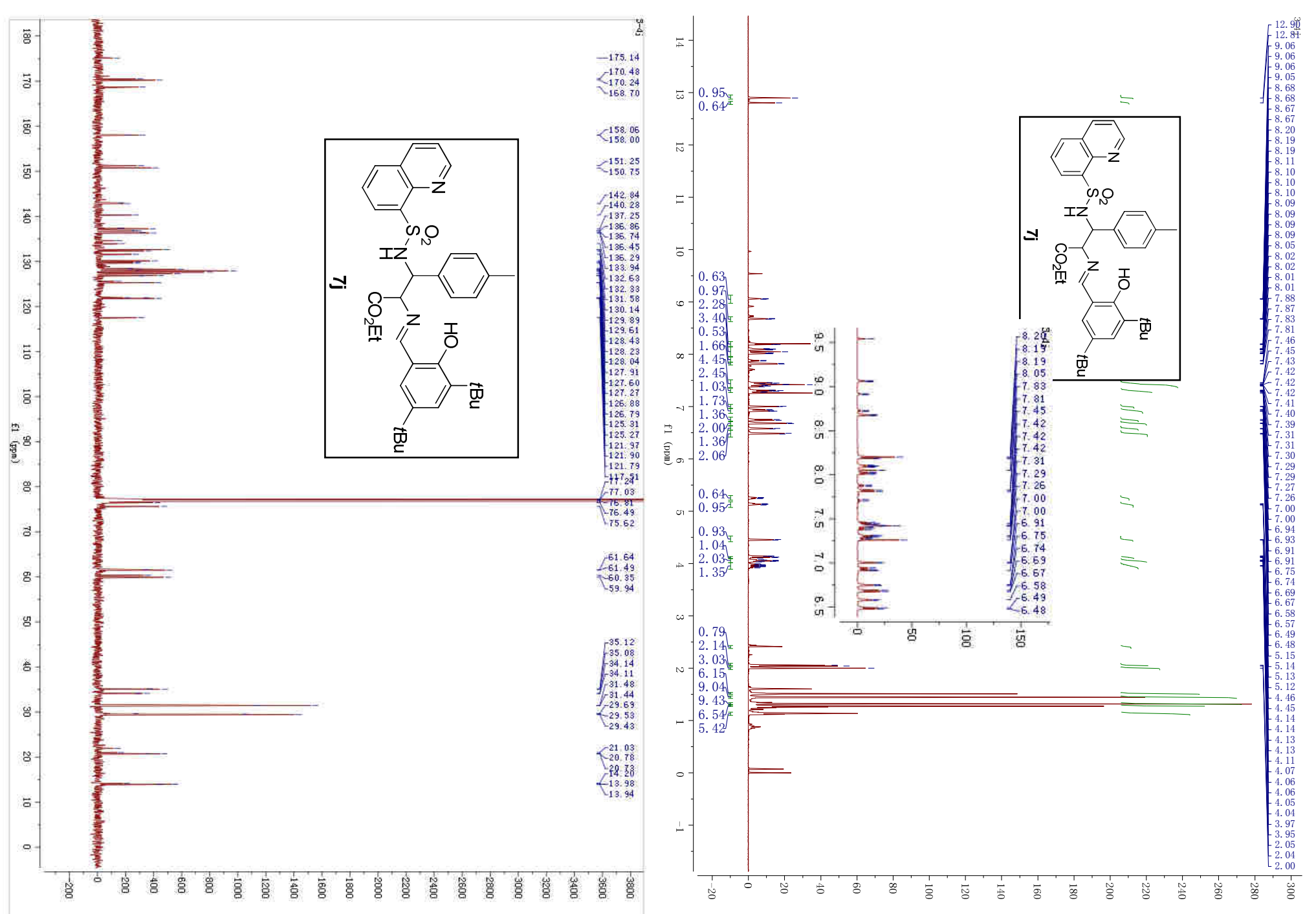


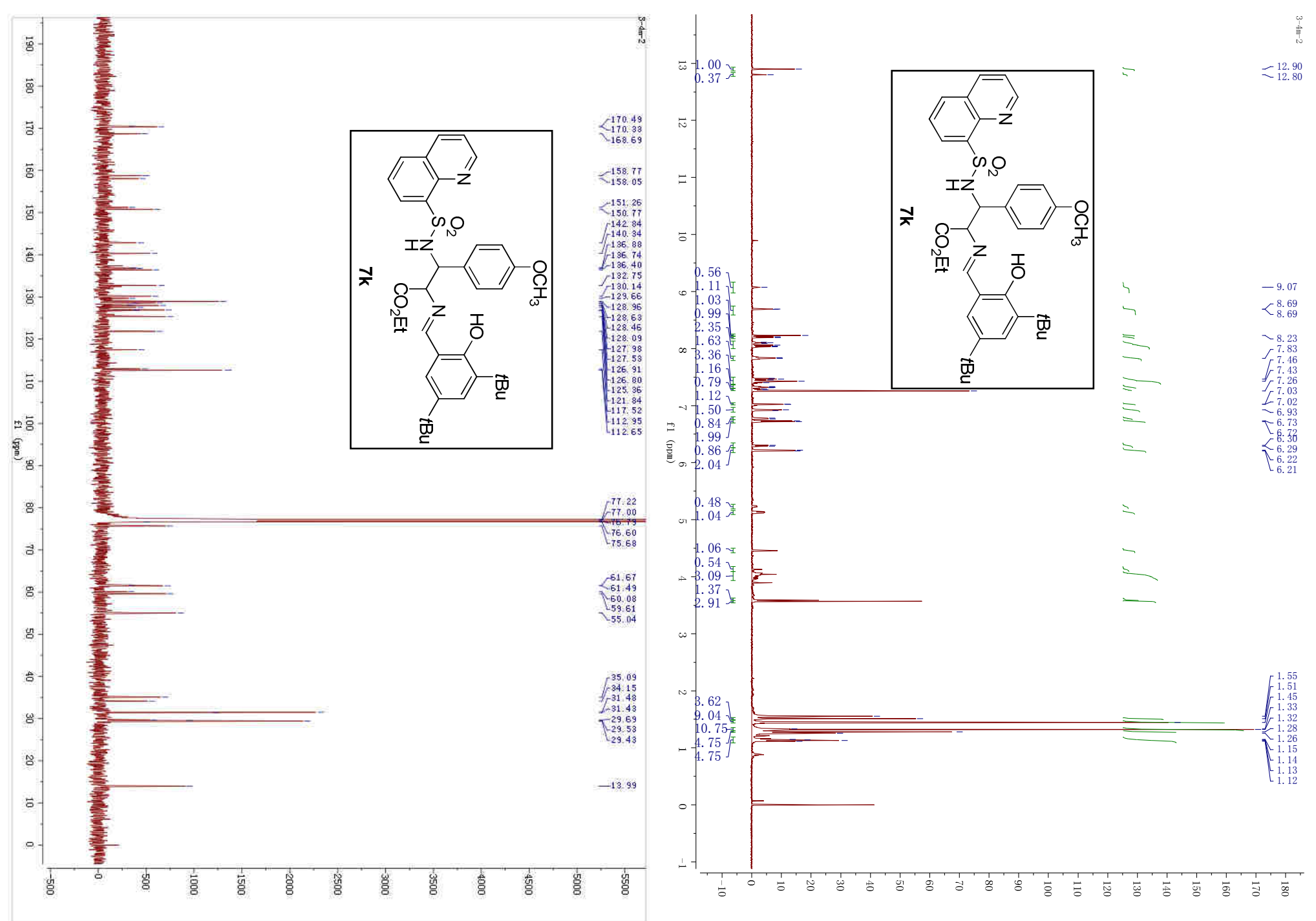



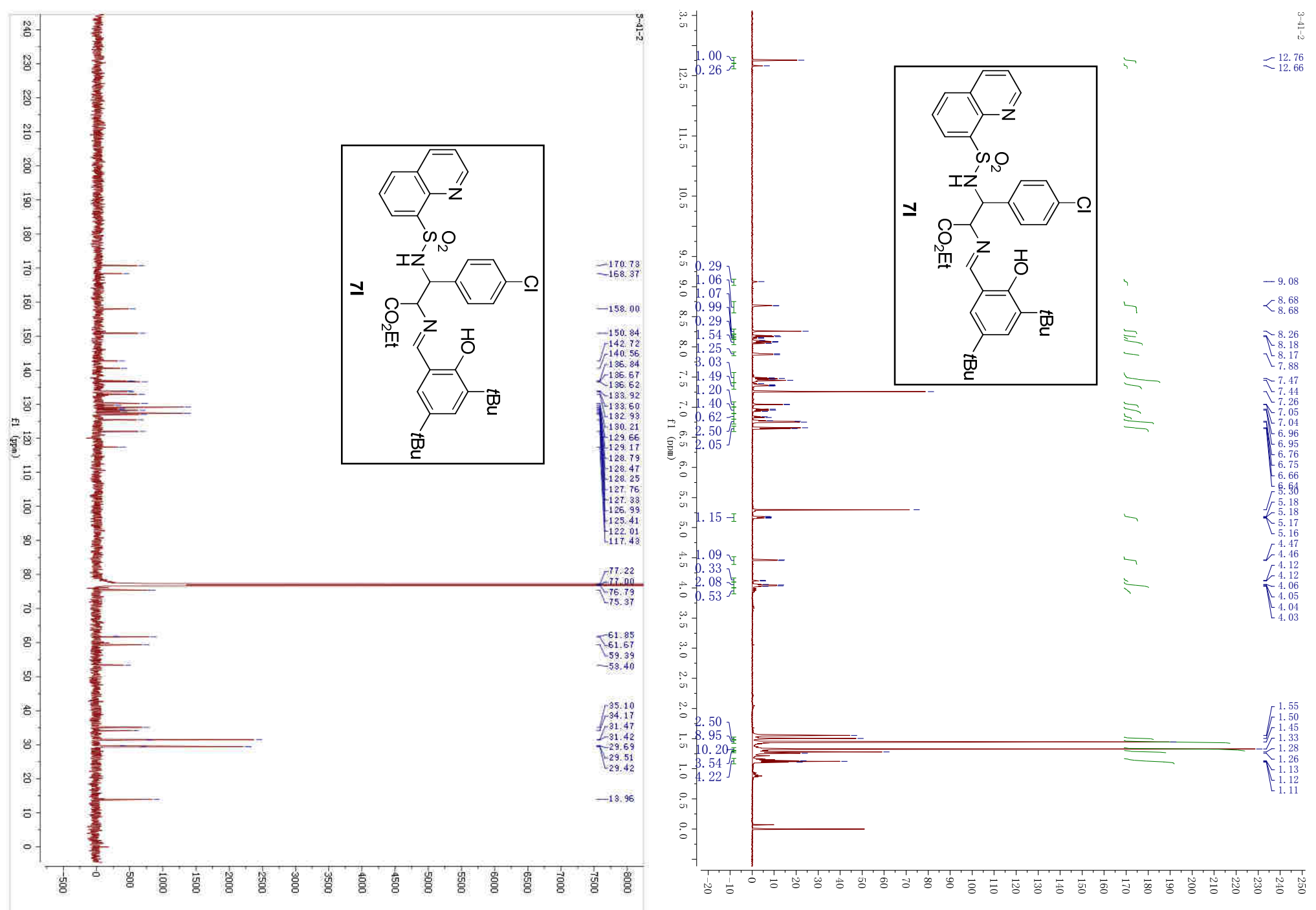


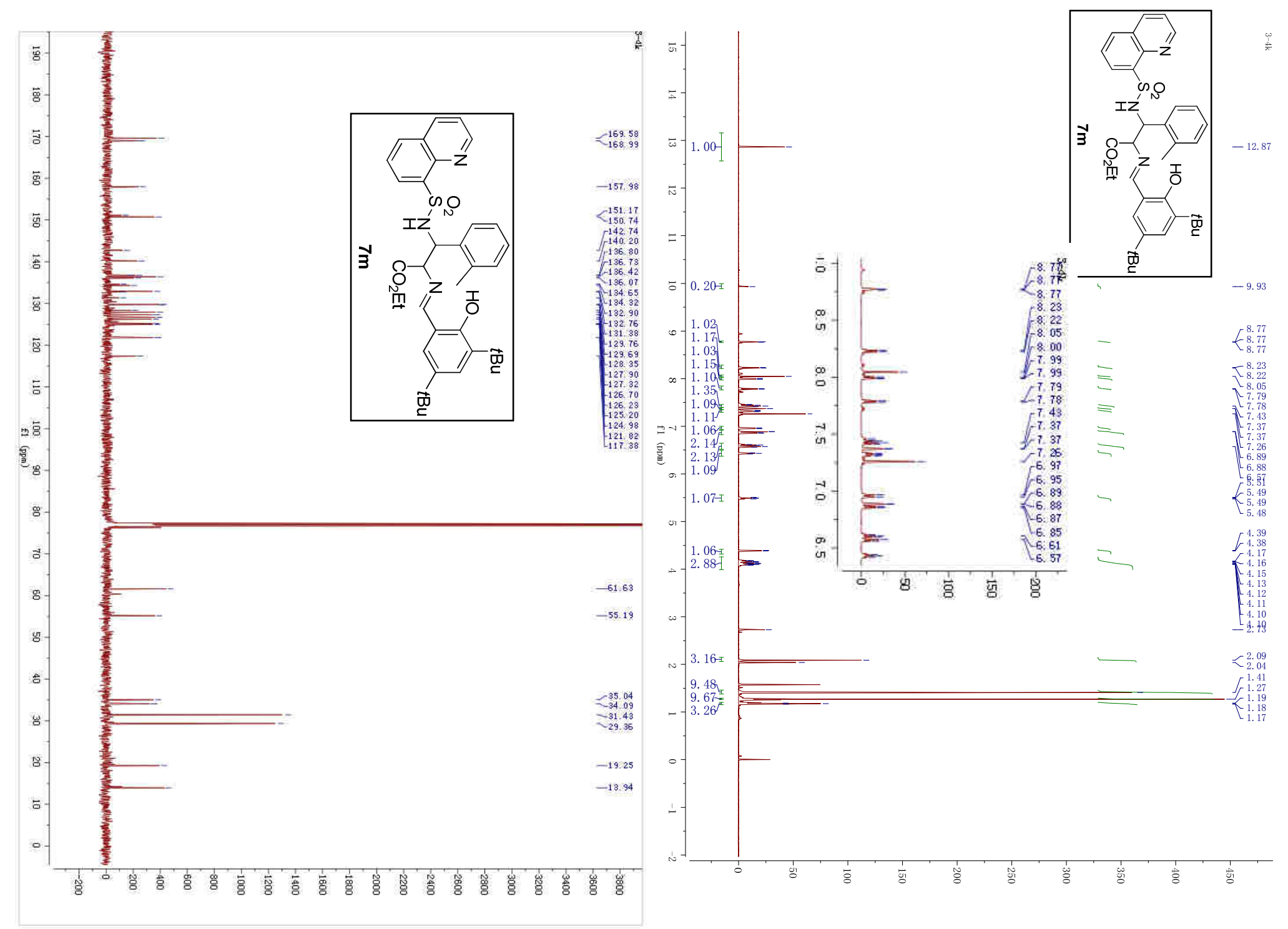




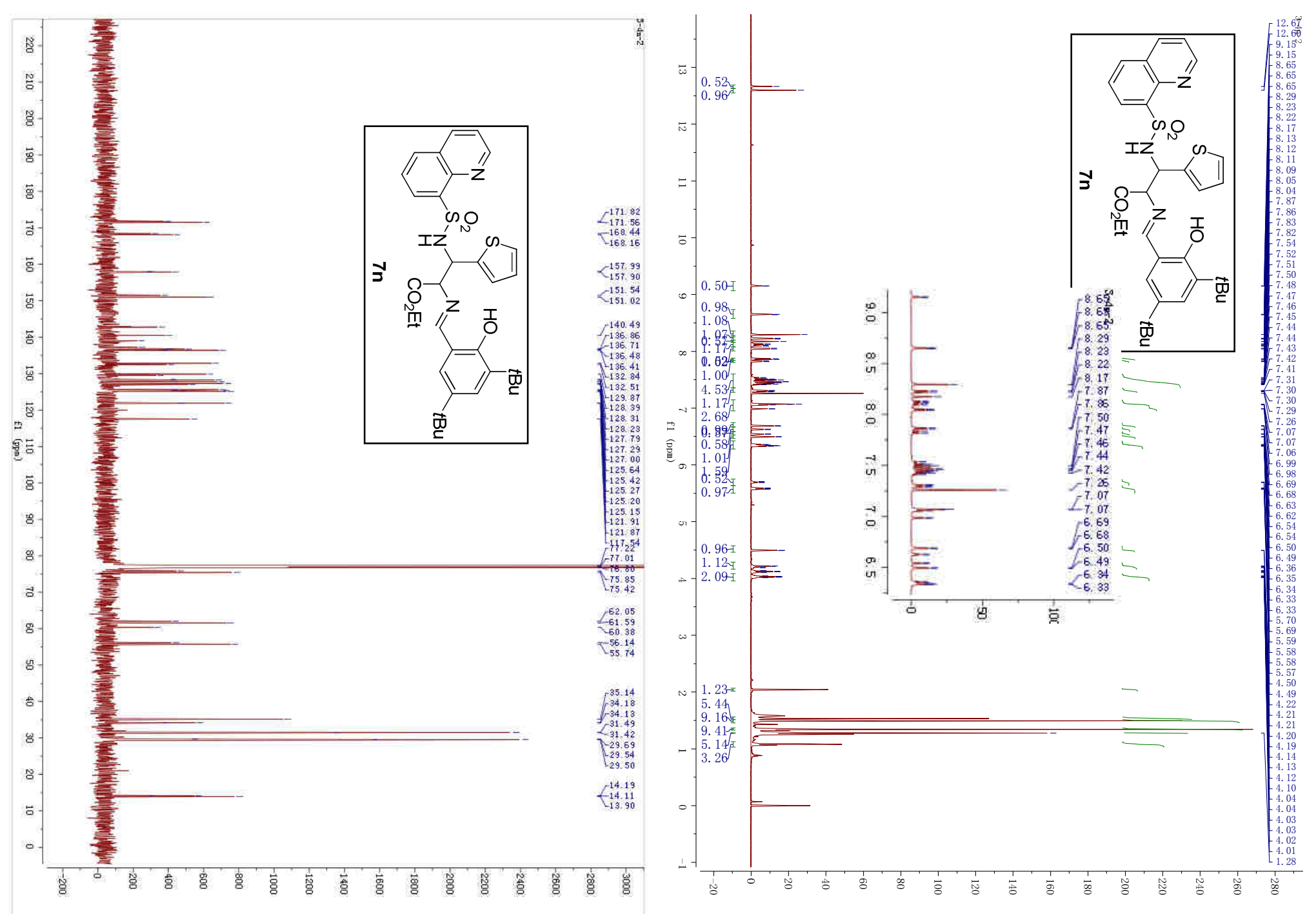




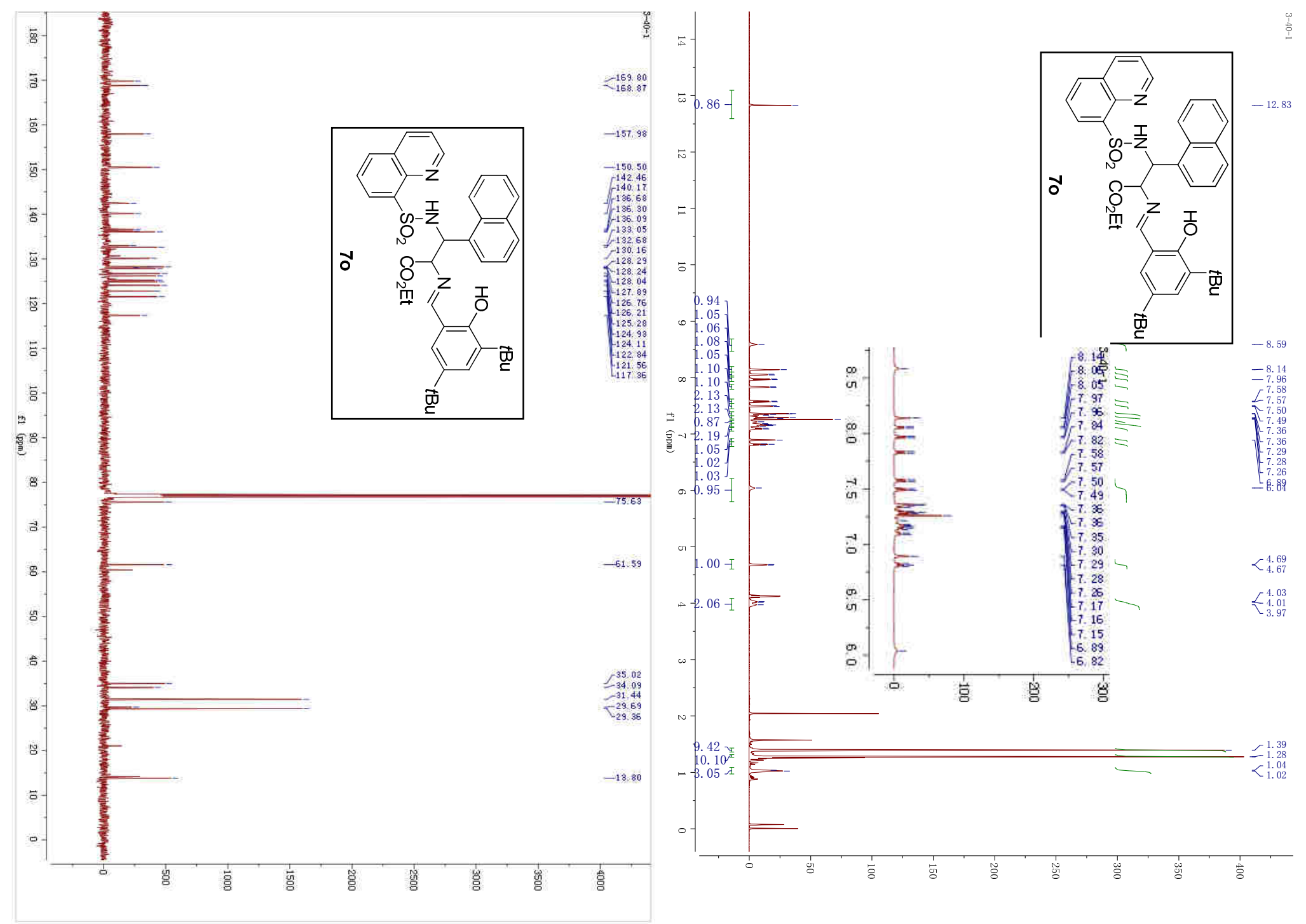



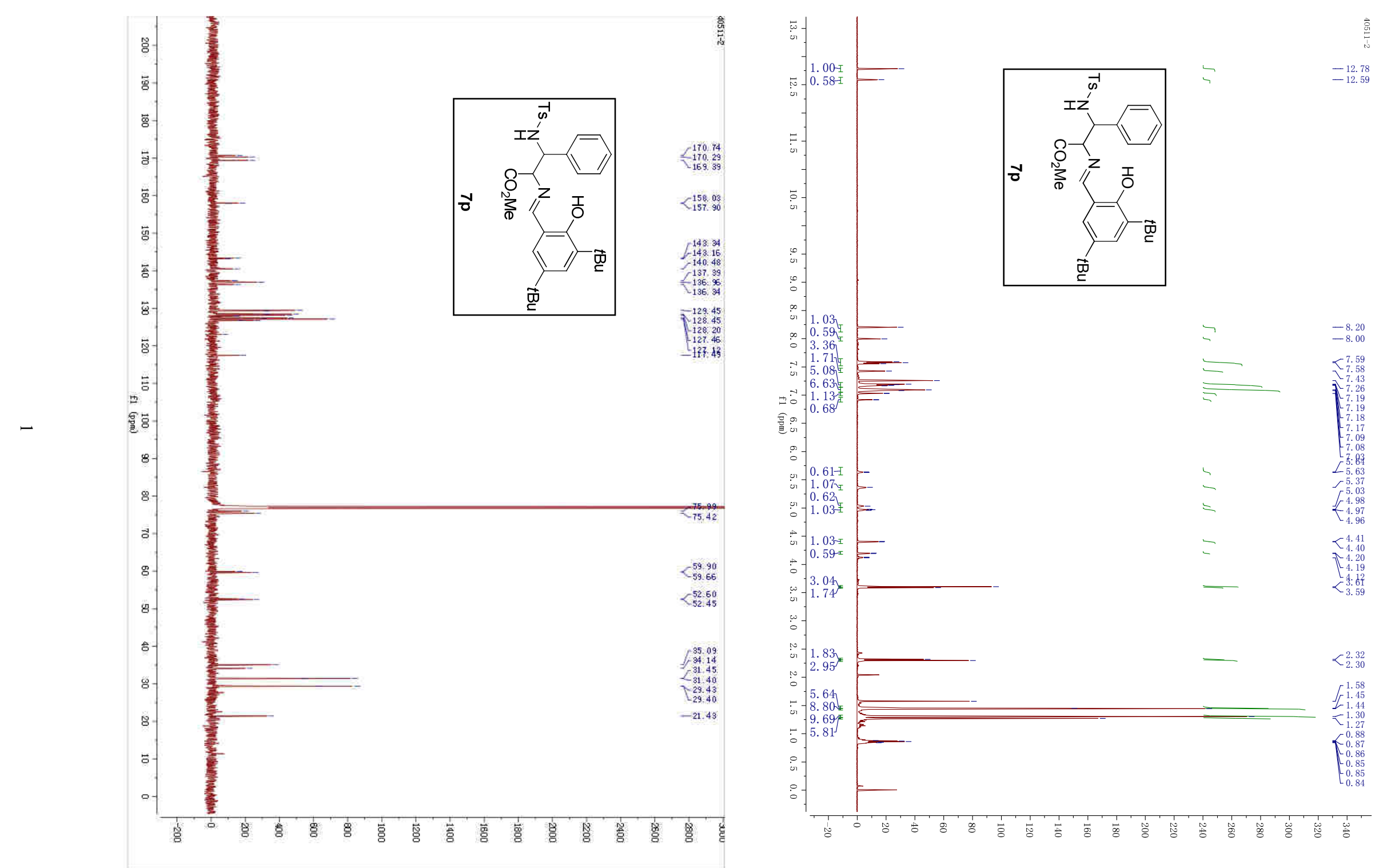
[1] M. Ayerbe, A. Arrieta, F. P. Cossio, A. Linden, Journal of Organic Chemistry 1998, 63, 1795.

[2] C. Palomo, M. Oiarbide, R. Halder, A. Laso, R. Lopez, Angewandte Chemie-International Edition 2006, 45, 117.

[3] J. Hernandez-Toribio, R. Gomez Arrayas, J. C. Carretero, Journal of the American Chemical Society 2008, 130, 16150.

[4] L. Wu, G. Li, Q. Fu, L. Yu, Z. Tang, Organic \& Biomolecular Chemistry 2013, 11, 443.

[5] H. M. Lovick, F. E. Michael, Tetrahedron Letters 2009, 50, 1016.

[6] L. Bernardi, M. Fochi, M. C. Franchini, A. Ricci, Organic \& Biomolecular Chemistry 2012, $10,2911$.

[7] T. Isobe, K. Fukuda, T. Ishikawa, Tetrahedron-Asymmetry 1998, 9, 1729.

[8] S. Dong, X. Liu, X. Chen, F. Mei, Y. Zhang, B. Gao, L. Lin, X. Feng, Journal of the American Chemical Society 2010, 132, 10650.

[9] X.-X. Yan, Q. Peng, Q. Li, K. Zhang, J. Yao, X.-L. Hou, Y.-D. Wu, Journal of the American Chemical Society 2008, 130, 14362. 